\title{
The Effects of Starbursts and Low-Surface-Brightness Galaxies on Faint Galaxy Models
}

\author{
B. A. McLeod \\ Harvard-Smithsonian Center for Astrophysics, 60 Garden Street, MS-20, Cambridge, MA 02138 \\ Email: bmcleod@cfa.harvard.edu \\ and \\ M. J. Rieke \\ Steward Observatory, University of Arizona, Tucson, AZ 85721 \\ Email:mrieke@as.arizona.edu
}

\begin{abstract}
We present models predicting the magnitude, redshift, and color distributions of field galaxies. We explore whether a fading starburst scenario can account for the observations of faint blue galaxies. We marginally rule out a starburst scenario with a local IMF because the models predict too many nearby faint galaxies that are not observed. A burst model with a truncated IMF reproduces the counts and redshift distributions well but produces too blue a population. We show that surface brightness selection has a significant effect on the distributions. In particular, adding a population of low-surface brightness galaxies, known to exist locally, can explain the counts for $B_{J}<23$. They do not, however, account for the steepness of the counts at fainter magnitudes. Finally we show that the colors of galaxies in a $K$-band selected sample are too blue to be consistent with a constant star-formation rate, passive-evolution model.
\end{abstract}

\section{Introduction}

A powerful test of our understanding of galaxy evolution and cosmology lies in understanding the observed properties of galaxies over as large a range of wavelengths and magnitudes as possible. We wish to create a model which specifies the basic cosmological parameters $\left(q_{0}\right.$ and $H_{0}$ ) and a description of the distribution of the physical properties of galaxies, i.e., their spectra, luminosities, and sizes. A complete description must also include the time-dependence of these properties. From this description we can then predict observable properties: the distribution of apparent magnitudes, colors, and redshifts. Since we cannot directly observe the evolution of individual galaxies, our understanding of evolution can be tested only through changes in the populations of galaxies. 
Hubble (1936) showed that the number of galaxies per magnitude interval, $n(m)$, followed the relation $d \log n / d m=0.6$ for bright galaxies, as predicted by simple theory. A detailed derivation of $n(m)$ as a function of $q_{0}$ was done by Sandage $(1961 ; 1988)$, who showed that, to first order, $n(m)$ is independent of $q_{0}$. The importance of galaxy evolution was shown by Brown \& Tinsley (1974) who pointed out that "a meaningful interpretation of the $N(m)$ relation will require good estimates of the luminosity functions, spectral energy distributions and evolution of a wide range of types of galaxies." However, the sophistication of the models to explain the observed distributions remained relatively simple until recently, partly due to a lack of observational constraints.

Early determinations (e.g. Peterson et al. 1979) showed that $n(m)$ in the blue had a slope consistent with moderate amounts of luminosity evolution. The interpretation that a steep slope implies luminosity evolution was made in the absence of redshift distributions, $n(z)$, and it came as a surprise to many when the excess galaxies turned out to be at fairly low redshifts (Broadhurst, Ellis, \& Shanks 1988). An additional puzzle came with deep $K$-band field counts (Cowie 1991; Gardner, Cowie, \& Wainscoat 1993), which showed no excess of galaxies over that predicted by a simple no-evolution model.

There have been several approaches to explaining the apparent discrepancy between the simple models and the observations. One possibility is the addition of a population of starbursting galaxies (Lilly, Cowie, \& Gardner 1991) with the number of bursting galaxies increasing with redshift. These galaxies have since faded, been disrupted or merged. Broadhurst, Ellis, \& Glazebrook (1992) addressed the merging scenario with a model that combined merging with substantial luminosity evolution to match the $K$ and $B_{J}$ counts and $B_{J}$ redshift distributions. However, it is not clear that the present population of galaxies can account for the required merger products (Dalcanton 1993). Yoshii (1993) has recently argued that introducing a non-zero cosmological constant is sufficient to bring the models into agreement. On the other hand, gravitational lensing statistics severely constrain the cosmological constant (Maoz \& Rix 1993). Koo \& Kron (1992) and Koo, Gronwall, \& Bruzual (1993) have argued that the local luminosity function is not sufficiently well known to rule out models with no merging and no new populations. They present a no-evolution model that fits a large range of observables, but at the expense of removing nearly all the luminous red galaxies from the local luminosity function. Continuing with this strategy, Gronwall \& Koo (1995) have added passive evolution to the models and further improved the quality of their fits. However, the starbursting galaxies in their models are still treated in an unphysical, no-evolution manner.

With such a complex problem, only a limited set of scenarios can be considered. Here we restrict ourselves to zero cosmological constant and we do not consider galaxy merging. We concentrate on evaluating the effects of adding a fading starburst population and considering surface-brightness selection effects. The evolutionary properties of the galaxies, including starbursts, are constrained by stellar-evolution models, i.e. no arbitrary luminosity evolution of galaxies will be allowed (e.g. Lilly 1993). We also try to evaluate each model with a large number of observational constraints. 
We begin with a brief description of the distributions to be computed. In $\S 3$. we present a compilation of previous observational results. Section 4. considers a simple model with a quiescently evolving population. The next section introduces starburst populations to examine their effects on the excess blue counts. This is followed by a discussion of surface brightness effects in $\S 6$. including the effects of known low-surface-brightness galaxies. We conclude with the implications that the $I-K$ colors of a $K$-selected sample have on evolution around $z=1$.

\section{Theory}

We consider three basic types of distributions: 1) number of galaxies as a function of apparent magnitude for a given filter; 2) number of galaxies as a function of redshift in a particular apparent magnitude range; and 3) number of galaxies as a function of color in a particular apparent magnitude range. The first quantity, the number-magnitude relation, is given by

$$
n(m)=\sum_{i} \int_{0}^{z_{f}} \phi_{i}(M(m, z)) \frac{d V}{d z} d z .
$$

The sum is over galaxies of different spectral types, $\phi_{i}$ is the luminosity function for galaxy type $i, z_{f}$ is the redshift the galaxies formed, and the volume element, $d V / d z$, is determined by the cosmology. The relation between apparent and absolute magnitude is given by

$$
M=m-5 \log \frac{d_{L}(z)}{10 \mathrm{pc}}-2.5 \log \frac{(1+z) \int_{0}^{\infty} S_{i}(\lambda, t(z=0)) F(\lambda) d \lambda}{\int_{0}^{\infty} S_{i}\left(\frac{\lambda}{1+z}, t(z)\right) F(\lambda) d \lambda}-\Delta_{i}(M, z),
$$

where $d_{L}$ is the luminosity distance, $S$ is the spectrum of the galaxy as a function of time, $F$ is the filter transmission, and $\Delta$ is the aperture correction. The third term, the KE correction, is due to two effects: the redshift of the galaxy shifts the observed bandpass to a bluer and narrower range of emitted wavelengths ( $k$-correction), and the spectrum of the galaxy changes with time (evolution). For now we will assume that $\Delta=0$, i.e., the observed magnitude measures the total flux from the galaxy. This will be discussed further below.

The number of galaxies in a given redshift and magnitude bin is

$$
n\left(z_{l}, z_{u}, m_{l}, m_{u}\right)=\sum_{i} \int_{z_{l}}^{z_{u}} \int_{m_{l}}^{m_{u}} \phi_{i}(M(m, z)) d m \frac{d V}{d z} d z .
$$

The color distributions are determined by performing the integrals over magnitude and redshift, but at each step of the integration the color of the galaxy is determined and the luminosity function value is added to the appropriate color bin. 


\section{Data}

Numerous observations of galaxy count vs. magnitude have been made. To obtain the best estimate of the number of galaxies at each apparent magnitude, we have combined the observations of several authors. Each data point is an average of the available data, weighted by the area surveyed. We present data only where the total number of galaxies observed is greater than 20. Data for $U, B_{J}\left(B_{J}=B-0.3(B-V)\right)$, Gunn- $r, I$, and $K$ are presented in Tables 1-5, respectively. The tables list the centers of the magnitude bins, the number of galaxies per square degree per magnitude, and the number of galaxies actually observed in each bin. The sources are drawn from the lists in the recent review by Koo \& Kron (1992) and the $K$-band summary by Gardner et al. (1993). Additional $K$ data are from McLeod et al. (1995). In some cases the data to be combined were made through somewhat different filters so we have applied the color transformations given by the authors. Note that the zero point for $r$ is based on an F-star spectrum (Thuan \& Gunn 1976) and so differs from the normal A0-star normalization in that $r$ is 0.43 mag larger than expected. The counts are from photographic surveys for $U<22, B_{J}<23.5$, $r<22.0$, and $I<21.0$; fainter counts are from CCD surveys.

\section{Quiescent Population}

\subsection{Spectral energy distributions}

We use the evolution models of Bruzual \& Charlot (1993) to produce galaxy spectral energy distributions (SEDs). In all cases we have assumed a Salpeter (1955) initial mass function (IMF), i.e, $d n(m) / d m \propto m^{-2.35}$. Each of the models consists of two components: an instantaneous burst at $\mathrm{t}=0$ plus a constant star formation rate (SFR) thereafter. By adjusting the ratio of the strength of the burst to the constant component we can affect the shape of the SED. The ratios were chosen so the models at an age of 13.5 Gyr would match the optical SEDs in Coleman, Wu, \& Weedman (1980) with a near-infrared extension (Rieke \& Rieke, in preparation) appended. Table 6 lists the amount of star formation per Gyr relative to the amount in the initial burst. Figure 1 shows these SEDs. The early-type galaxies are completely dominated by the initial burst; the late-type galaxies are dominated by the constant component. Figure 2 shows a comparison of the expected counts using the Bruzual SEDs vs. the Coleman et al. SEDs. This Bruzual model assumes that there is no evolution of the SEDs with time, an unphysical assumption, but necessary for this purpose because the Coleman et al. SEDs contain no evolutionary information. The purpose of this figure is to illustrate the order of magnitude of the errors in the counts due to uncertainties in the SEDs. Most of the divergence occurs in the faint blue counts where the lack of understanding of the ultraviolet spectra of galaxies becomes important. 


\subsection{Luminosity functions}

We initially consider two different local luminosity functions (LFs). The first set, used by Lilly (1993), are the type-dependent LFs presented by Bingelli, Sandage, \& Tammann (1988), slightly adjusted to sum to a Schechter function with parameters $\phi^{*}=0.00175 \mathrm{Mpc}^{-3} \mathrm{mag}^{-1}$, $M_{B_{J}}^{*}=-21.0$, and $\alpha=-1.15$. The Schechter function (Schechter 1976) is defined by $\phi(M)=0.92 \phi^{*} \exp \left\{-0.92\left(M-M^{*}\right)(\alpha+1)-\exp \left[-0.92\left(M-M^{*}\right)\right]\right\}$. This total LF is the one derived by Loveday et al. (1992), but with a slightly steeper faint end slope. All LF specifications in this paper are scaled to $H_{0}=50 \mathrm{~km} \mathrm{~s}^{-1} \mathrm{Mpc}^{-1}$ for easy comparison with other authors. We pair each morphological type LF with the corresponding Bruzual SED. The second luminosity function we consider is the color-dependent derivation by Shanks (1990). This LF is divided into three classes, $B-V<0.65,0.65<B-V<0.85$, and $B-V>0.85$. We further arbitrarily divide each class equally among the SEDs which fall within that color range, as shown in Table 6. The overall normalization of the LFs is chosen so as to match the observed counts at $B_{J}=17$. Figure 3 shows a comparison of the resulting color distributions of bright $\left(15<B_{J}<17\right)$ galaxies from the two LFs along with the observed distribution presented in Koo \& Kron (1992). The Lilly LF produces too few blue galaxies relative to red ones, while the Shanks LF has a more correct distribution. Ideally we would like a LF with more than three divisions according to color, but for now we will adopt the Shanks local LF.

\subsection{Results}

In Figure 1 we show the resulting counts through the $U, B, r, I$ and $K$ filters. From above, we assume that the present day age of the quiescent population is $13.5 \mathrm{Gyr}$. The value of $H_{0}$ was chosen so that the galaxies are not formed at too low a redshift. For example,

$H_{0}=50 \mathrm{~km} \mathrm{~s}^{-1} \mathrm{Mpc}^{-1}$ puts the redshift of formation at $z=2.5$, which would be excluded by the redshift distributions (galaxies in their initial burst would be seen at relatively bright magnitudes). We have adopted a redshift of formation, $z_{f}$, of 7.3 and assume $q_{0}=0.05$, which leads to a choice of $H_{0}=60 \mathrm{~km} \mathrm{~s}^{-1} \mathrm{Mpc}^{-1}$.

This baseline model (solid lines) shows the same basic feature of galaxy count models made by various previous authors: the simple model matches the $K$-band counts reasonably well, but drastically underestimates the faint blue counts. All the models show a significant discrepancy with the bright-end $r$ observations. The reason for this is not clear. One possibility is that our SEDs are somehow drastically wrong in the $r$ band. This seems unlikely since the Coleman et al. SEDs in Figure 2 produce the same effect. Secondly, the observations could have a zero-point error; or it could be a true large scale structure effect. Picard (1991) sees 30\% differences between his northern and southern fields which he attributes to structure. His observations are a factor of 
two higher than those of Sebok (1986). Recent results from the second Palomar Sky Survey (Weir, Djorgovski, \& Fayyad 1995) are intermediate between Picard and Sebok. These discrepancies will require further work to resolve.

The dashed line of Figure 4 shows the same evolutionary model for $q_{0}=0.5$. To preserve the present day SED ages of 13.5 Gyr with $z_{f}=7.3$ we have changed $H_{0}$ to 47 . The only effect of changing $H_{0}$ in these models is to change the relation of age to redshift. Figure 5 shows $B_{J}$-selected redshift distributions from Koo, Gronwall, \& Bruzual (1993) with the predictions of the $q_{0}=0.05$ model superimposed. As in Koo et al. (1993), the histograms are shown in seven logarithmic bins per decade. Unlike other authors, we have normalized the observed redshift distributions to match the observed total number of galaxies in each magnitude interval rather than the predicted number. The ratio of the area under the histogram to that under the model curve in each panel should correspond to the prediction in the $n(m)$ plot. Thus it is easy to see from the redshift distributions that the model underpredicts the number of galaxies present for faint $B_{J}$.

\subsection{Alternate inputs}

Many different star formation histories can give rise to a given present day SED. We have also explored using exponential SFRs to produce the SEDs and find that they produce too much luminosity evolution. Specifically we used the exponential models adopted by Gronwall \& Koo (1995), including reddening, combined with the luminosity function adopted above. This exponential SFR model overpredicts, by factors of several, the number of galaxies with $B_{J} \approx 22.5$ and $z>0.8$. The model of Gronwall \& Koo has no high redshift excess because their derived luminosity function drastically reduces the number of bright galaxies of intermediate spectral type compared with the derived LFs of Marzke, Huchra \& Geller (1994) and of Shanks (1990). We thus choose to use the burst-plus-constant form of the SFR and limit the amount of luminosity evolution. It is worth remembering though that observationally derived LFs may have significant errors at the extreme bright end, so this topic warrants further investigation. Exponential star-formation is revisited in $\S 7$. when we discuss colors of $K$-selected galaxies.

We have also considered the effects of replacing our color-dependent LF with the typedependent LF of Marzke et al. (1994). This LF has a more steeply rising faint end than the one we adopted. We find that as with the Lilly (1993) LF, we get a bright population that is somewhat too red. This is likely due to problems making a one-to-one association of mophological types with color, and a more careful treatment may give better results. However, a comparison of $n(m)$ between the two LFs reveals that they give nearly identical results. This gives us confidence that minor changes in the LF do not dramatically change our results. 


\section{Starburst Populations}

In this section we consider the effects of making up for the deficiency of faint blue galaxies by adding a population of starburst galaxies. We assume that each new galaxy has a single burst of star formation lasting 0.1 Gyr and thereafter fades quiescently. This type of population should provide the largest amount of fading possible and test whether such galaxies can account for an excess of blue galaxies. For computational reasons we add the bursts at discrete points in time, one burst population for each of the observed redshift distribution bins.

The luminosity functions of the burst galaxies are determined empirically. By subtracting the baseline-model redshift distributions from the observed distributions we get the number of excess galaxies in each bin. A further division by the comoving volume of the redshift bin converts to a spatial density and one more normalization corrects for the fact that the burst galaxies are in their bright phase for only a portion of the time represented by the redshift bin they are in. We assume an effective lifetime of the burst phase of 0.2 Gyr, i.e the time for the stars to form and fade by a factor of $e$. The KE-correction and distance modulus determine the peak absolute magnitudes required to produce the observed apparent magnitudes. The characteristics of the burst population are tabulated in Table 0 and the derived burst rate LFs are shown in Figure 6 . There are no obvious trends of the derived LFs as a function of redshift. A fit done by eye shows that Schechter parameters of $B_{J}^{*}=-19.0, \phi^{*}=0.02 \mathrm{Mpc}^{-3} \mathrm{mag}^{-1} \mathrm{Gyr}^{-1}$, and $\alpha=-1$ are a reasonable fit to the ensemble of LFs. Here we have normalized the amplitude $\phi^{*}$ in terms of the rate of burst formation per Gyr. Note that since the absolute magnitudes are measured at the brightest phase, a galaxy with $M_{B_{J}}=-19.0$ has a mass of $2 \times 10^{8} M_{\odot}$, a factor of 100 less massive than an old galaxy of the same luminosity would have.

Figures 019 (solid lines) show the magnitude, redshift, and color distributions for a burst model where the bursts have an IMF identical to the baseline population (Salpeter, $0.1-125 M_{\odot}$ ). Now the blue counts fit reasonably well up to $B_{J}=24$. They turn over at fainter magnitudes because we have no redshift distributions at fainter levels and have made no attempt to extrapolate the burst population. The most serious concern is the redshift distributions. Although the high- $z$ end of each distribution fits well, this model predicts a low- $z$ tail in the $B_{J}>20$ distributions that is not seen in the observations. This effect is due to the fact that the low-mass stars are long-lived and so the galaxy never fades completely. This effect is under-represented in this model since we have not added $z>1$ bursts. In $\S 6$. we will consider whether surface brightness selection effects can reduce the low redshift tail. One may also question how reliable the observations are. Tresse et al. (1993) find an excess of low-redshift galaxies in an $I$-band selected redshift survey. However, this survey contains a small number of galaxies. On the other hand, Glazebrook et al. (1995) argue that a low-redshift excess is unlikely, based on their $22.5<B<24$ redshift survey.

They find no excess over the baseline model. These observational discrepancies will become easier to resolve in the next few years with new multi-object spectrographs on large telescopes.

The $B_{J}-R_{F}$ color distributions now show an excess at the blue end but still a deficit at the 
red end. This could be alleviated by either adding reddening to the models or adding an older stellar component to the galaxies. However, both of these would exacerbate the problem of the low-redshift tails. Adding an older stellar component would increase the amount of light that remains luminous to the present. Adding dust would require that, to maintain the same observed blue luminosity at high redshift, we must increase the total luminosity of the burst. The net result would again be to decease the amount of fading and increase the low-redshift population. In reality, both dust and old stars are likely to be present, and an improved model would take these into account.

Modeling of the stellar populations of the nearby starburst galaxy M82 (Rieke et al. 1993) suggests that while the slope of the IMF in M82 may be equal to that of the local IMF, very few low-mass stars are produced. We now consider a starburst model using a Salpeter IMF but truncated so that no stars with $M<2.5 M_{\odot}$ are produced. An application of the Bruzual \& Charlot (1993) models to this type of IMF is presented by Charlot et al. (1993). The LFs derived are identical to the ones above, but because the burst fades so rapidly, we assume that the effective bright lifetime of the burst is the same as the burst length, 0.1 Gyr. Thus the LFs have a normalization twice as large as previously. For this IMF a $M_{B_{J}}=-19.0$ galaxy has a mass of $6 \times 10^{7} M_{\odot}$. The amount of fading ranges from $15 \mathrm{mag}$ after $2 \mathrm{Gyr}$ to $19 \mathrm{mag}$ after $4.3 \mathrm{Gyr}$. After 5 Gyr the galaxy has faded completely because all the stars have turned into stellar remnants.

Figures 79 (dashed lines) show the distributions for this model. As in the previous burst model, the $U$ counts are over predicted. The redshift distributions this time are better, having no tails at low redshift. The reason for this is clear: the galaxies, having no low-mass stars, fade completely and so are undetectable except in their burst phase.

\section{Surface Brightness Effects}

Up to now we have assumed that all galaxies of a given apparent magnitude will be detected. In practice, a galaxy will be detected only if its observed surface brightness is above a threshold value $\left(\mu_{d e t}\right)$ over a minimum angular area $\left(\mathrm{A}_{\text {min }}\right)$. The values of $\mu_{\text {det }}$ and $\mathrm{A}_{\text {min }}$ are different for each survey. The observed surface brightness profile depends on the intrinsic galaxy profile convolved with a point spread function. In the detection process, the image is often further convolved with a smoothing function. We use the formalism developed by Yoshii (1993) to compute the detectability of galaxies. The detected magnitude of a galaxy differs from the total integrated magnitude by $\left.\Delta=m_{\text {det }}-m_{\text {tot }}=-2.5 \log \left(\tilde{G}\left(\theta_{\text {det }}\right) / \tilde{G}(\theta)\right)\right)$, where $\tilde{G}(\theta)$ is Yoshii's notation for the integrated intensity out to radius $\theta$. The expression for $\tilde{G}$ depends on a galaxy's intrinsic profile, its redshift, and the seeing conditions. The value of $\theta_{\text {det }}$ is fixed for an aperture magnitude, and for an isophotal magnitude, again depends on the galaxy's properties. Thus $\Delta$ is a function of galaxy type, $M, z$, and the detection conditions. To compute Eq. 1 and 3 we first 
iteratively solve Eq. 2 for $M$, then determine whether the galaxy survives the detection criteria before evaluating the LF.

Table 8 lists the surface brightness selection criteria we adopt for the redshift distributions. We have tried to take from the literature the parameters used to produce the observed distributions. However, often the information given in the papers is incomplete and we have made reasonable guesses for the missing parameters. In some cases the redshifts come from several sources with different selection criteria. Thus the precise quantitative effects of the selection criteria should not be taken as absolute, but we believe that the effects shown are quite representative.

\subsection{Quiescent population}

For our quiescent population we assume that the E and S0 types have an $\mathrm{r}^{1 / 4}$ profile where the central surface brightness, $\mu_{0}$ at $z=0$ is assumed to be $14.8 B_{J}$ mag $\operatorname{arcsec}^{-2}$ (Fish 1964). For the spirals we assume an exponential profile with $\mu_{0}=21.0$ for types Sab and Sc, and $\mu_{0}=22.2$ for Sdm and Irr (van der Kruit 1987). These are the mean apparent central surface brightnesses for these galaxy types, rather than a value corrected for inclination. This is appropriate because in our calculation we make no consideration of galaxies of different inclinations. Van der Kruit's results are fully consistent with those of Freeman (1970) who obtained $\mu_{0}=21.7$ averaged over all types after inclination correction.

\subsection{Burst population}

For the burst population we make the same assumption with regards to central surface brightness as for the quiescent population: galaxies of the same age have the same central surface brightness for all magnitudes. Figure 10 shows the predicted redshift distributions for three scalings: for a $10^{8} M_{\odot}$ burst the galaxies have half-light radii $\left(r_{e}\right)$ of 1 (solid), 3 (dashed), and $10 \mathrm{kpc}$ (dotted).

In the $B_{J}=21-23$ bins, the low-redshift tails are strongly dependent on the sizes of the burst galaxies. With the normal IMF, a $10^{8} M_{\odot}$ burst corresponds to an absolute magnitude of $M_{B_{J}}=-18$ during the bright phase. High-resolution imaging of a sample of galaxies with $21<B_{J}<22.5$ (Colless et al. 1994) shows that such a galaxy should have $r_{e} \approx 2 \mathrm{kpc}$. This corresponds to between the dashed and solid lines of Figure 10, at a level where the low-redshift tails are still in conflict with the observations. It is worth noting that the effects of surface brightness selection are different in each of the redshift bins. This points out the importance of understanding the selection criteria for each survey. 


\subsection{Low surface brightness galaxies}

Disk galaxies with low surface brightness (LSB) are known to exist in the local universe (McGaugh 1992) but are not included in the luminosity functions derived from large area photographic surveys (Impey 1993). A derivation of the luminosity function of these galaxies is not yet available but they are believed to exist in numbers comparable to the high surface brightness galaxies. McGaugh (1994) has argued that these LSB galaxies are identical to the faint blue galaxies that are missing from the baseline model. We now add such a population to our model. The evolutionary history of such galaxies is not well understood (McGaugh \& Bothun 1994) so for simplicity we assume a single SED of constant star formation with age 5 Gyr. This SED has colors $U-B=-0.18, B-V=0.33$, and $V-I=0.96$, chosen to match the median colors of the LSB galaxies, $U-B=-0.17, B-V=0.44$, and $V-I=0.89$ (McGaugh \& Bothun 1994). We will assume a single luminosity function identical to that for high surface brightness galaxies: $\phi=0.00175 \mathrm{Mpc}^{-3} \mathrm{mag}^{-1}, \alpha=-0.97, M_{B_{J}}^{*}=-21.0$ (Loveday et al. 1992). Additionally we assume no evolution for the LSB SED. By this we do not imply that these galaxies do not evolve, but we assume that the population as a whole does not change with time. An exponential surface brightness law with $\mu_{0}=23.5$ is adopted for all the LSB galaxies. These assumptions will certainly be subject to revision as more information on the LSB galaxy population becomes available.

Figure 11 shows that adding the LSB population has its strongest effect on the $B_{J}$ counts between 18 and 23. The nearby galaxies are not affected greatly because the surface brightness threshold in the local survey (Maddox et al. 1990b) is too high to detect many of them. For those that are detected, the extrapolation to pseudototal magnitudes (Maddox et al. 1990a) underestimates the actual magnitude by up 1 mag. At intermediate magnitudes the increased sensitivity of the surveys allows them to be detected. However, at high redshift in the absence of evolution, the galaxies once again are too faint to detect. The known population of LSB galaxies can explain the observations only for $B_{J} \lesssim 23$. A recent analysis of LSB galaxies by Ferguson \& McGaugh (1995) reaches a similar conclusion.

\section{K-selected colors}

Galaxies selected at $K$-band are a powerful probe of higher redshifts because of the smaller KE-corrections at longer wavelengths. Figure 12 shows the color distributions of galaxies from the sample described by McLeod et al. (1995). Overplotted (solid lines) is the predicted distribution from the baseline population. It is immediately clear that in the $K=19-20$ range, the model predicts colors that are too red. From Figure 13 we see that the predicted red galaxies are high-redshift objects with low amounts of star formation. The spikes in the $R-K$ and $B_{J}-K$ 
distributions are caused by the fact the model contains a discrete number of galaxy types and the color curves are flat at high redshift. In reality these distributions will be smoothed out. A particularly important color to consider is $I-K$ because even at redshifts approaching 2 , the observed $I$ band is still emitted longward of $3000 \AA$ where we have fairly good knowledge of what zero-redshift SEDs look like. The blue colors of the galaxies suggest two possibilities. The first is that the galaxies in the $K=19-20$ range are not at such high redshift. This would be the case if significant numbers of galaxy mergers occurred since redshift $1-2$ and we are seeing the galaxies before merging.

The second possibility is that the star-formation rate of present-day red galaxies was significantly higher at $z \approx 1$. We now replace the $\mathrm{E}$ and $\mathrm{S} 0$ populations from the baseline model with an exponentially decaying star-formation rate SED with $\tau=1 \mathrm{Gyr}$. The Sab SED is replaced with $\tau=2$ Gyr. For comparison with Table 6 these SEDs have present day (age = $13.5 \mathrm{Gyr}$ ) $B-V$ colors of 0.91 and 0.87 respectively. At $z=1.5$ the observed colors are considerably bluer than their constant-SFR counterparts with $I-K=3.8$ and 3.25 respectively. The dotted curves in Figure 12 show a better agreement with the observations than the baseline model. However, the $B_{J}$ redshift distributions now predict too many high-redshift galaxies compared with the observations (Figure 14).

The definitive test to distinguish between merging and an increased SFR requires determining redshifts for $K$-selected galaxies. Elston (1994) has reported results of a redshift survey of galaxies with $K<18$ and $R<22$ that shows a median redshift $>0.5$, implying that mergers are relatively unimportant at low redshift. In contrast, a partially complete survey to $K=20$ (Songaila et al. 1994) shows that the majority of galaxies are not at high redshift. Additional surveys to $K=19-20$ are currently underway in the UK and Arizona. The discrepancy between the baseline model and the observations becomes most apparent in the $K=19-20$ range, but determining complete samples of redshifts here will be quite difficult as a majority of the galaxies have $B_{J}>24$. The high-redshift galaxies, unfortunately, will be the most difficult to get redshifts for as the $k$-correction makes them the reddest.

\section{Summary}

We have explored several scenarios for explaining the magnitude, redshift and color distributions of galaxies. The baseline population of galaxies consists of an initial burst of star formation, followed by constant star formation thereafter. We conclude

1. The baseline population with passive evolution and the Shanks (1990) color-dependent luminosity function underestimates the number of galaxies at faint $B_{J}$. 
2. Adding a population of starburst galaxies to increase the counts at $B_{J}$ adds too many low-redshift remnants not seen in the $z$ distributions. The red stars in the starburst galaxies do not fade enough to be absent from local surveys.

3. Changing the IMF of the burst population removes the low-redshift tail problem. Both starburst populations produce galaxies that are too blue in $B_{J}-R_{F}$. Alleviating the blueness by adding dust or old stars would increase the low-redshift excess problem.

4. Adding surface brightness selection to the simulation has significant effects on the $n(m)$ and $n(z)$ relations at all magnitudes as previously discussed by Yoshii (1993). The low- $z$ tails produced by the local IMF starburst population are diminished but not eliminated.

5. Adding a population of low surface brightness galaxies, which are known to exist locally, removes much of the discrepancy in the counts brighter than $B_{J}=23$. At fainter magnitudes there is still a problem. Full understanding of this effect will require LFs corrected for surface brightness effects.

6. The blue $I-K$ colors of galaxies selected with $K=19-20$ show that an old quiescent population is not an adequate model for elliptical galaxies. Making the galaxies bluer can be accomplished by having them at $z=1$ but forming more stars. This appears to be ruled out by the $B_{J}$ selected redshift distributions. The alternative is to increase the number of lower redshift galaxies. They are bluer because of the $k$-corrections. The physical mechanism to accomplish this is not clear, though one possibility is for these galaxies to be in a premerged state.

One of the most important next steps in modeling galaxy distributions will be making use of surface brightness corrected luminosity functions to model the local population of galaxies. The analysis of a LSB population in $\$ 6.3$. is an important first step, but a more accurate treatment should be possible soon using recently derived luminosity functions for LSB galaxies (Sprayberry, 1994). The results of $\$ 6.3$. suggest that at higher redshift a starburst population will still be necessary. In the present models we considered the extreme situation of a given galaxy having only a single burst in its life. The result showed that with a normal IMF, these bursts leave remnants that should be detected nearby but are not accounted for in the local LF. Using a truncated IMF allows the galaxies to fade sufficiently. However, these model burst populations are all too blue in their $B_{J}-R_{F}$ colors implying that the observed galaxies must also have an older red population. Wyse \& Silk (1987) have argued that such bimodal star formation may have occurred in the solar neighborhood. Unfortunately, the presence of an old population exacerbates the lack-of-fading problem. Further analysis will be required to evaluate this scenario.

Closely coupled to the issue of starbursts is that of interactions and merging (Larson \& Tinsley 1978; Carlberg \& Charlot 1992). Incorporating merging into the modeling problem is complicated because an interaction changes the total number of galaxies, the amount of light (through induced star-formation) and potentially the scale-lengths of the light. Yoshii (1993) has 
suggested that pre-merger galaxies will not be detected due to surface brightness selection effects. In particular he argues that premergers cannot account for the number of galaxies required to make an $\Omega=1$ cosmology fit the $B_{J}$ and $K$ counts as is suggested by Broadhurst et al. (1992).

The starburst treatment that we have introduced is a good starting approximation to increased merging in the past. Our starburst galaxies represent the post-merger galaxies. An useful addition to the modeling program will be to add the number evolution associated with merging to the existing code. This will be especially important in investigating the apparent inconsistencies with the passive evolution model and the colors and redshifts of faint $K$-selected samples.

Another current deficiency is the state of the $n(m)$ data. Tables 15 are compiled from over two dozen separate sources, each with its own detection criteria, which are often not stated completely. To make matters more complicated, many different filters have been used. With large format CCD mosaics becoming available it is worth considering redoing large parts of the observations in a consistent manner. The ideal survey would image the same areas of the sky in multiple filters, providing color information for a large number of galaxies, and reducing discrepancies introduced by large scale structure. At the same time, the surface brightness selection criteria could be done in a consistent way, thus eliminating some of the difficulties in analyzing published data sets.

We would like to thank G. Bruzual for providing his galaxy evolution software, C. Gronwall and D. Koo for providing their color and redshift data, and G. Bernstein for many useful discussions. This work fulfilled part of the dissertation requirements of B.A.M. at the University of Arizona and was supported through an NSF Faculty Award for Women. 


\section{REFERENCES}

Bingelli, B., Sandage, A., \& Tammann, G. A. 1988, ARA\&A, 26, 509

Broadhurst, T. J., Ellis, R. S., \& Glazebrook, K. 1992, Nature, 355, 55

Broadhurst, T. J., Ellis, R. S., Shanks, T. 1988, MNRAS, 235, 827

Brown, G. S., \& Tinsley, B. M. 1974, ApJ, 195, 555

Bruzual A., G., \& Charlot S. 1993, ApJ, 405, 538

Carlberg, R. G., \& Charlot, S. 1992, ApJ, 397, 5

Charlot, S., Ferrari, F., Mathews, G. J., \& Silk, J. 1993, ApJ, 419, L57

Ciardullo, R., ApJ, 321, 607

Coleman, G. D., Wu, C.-C., Weedman , D. W. 1980, ApJS, 43, 393

Colless, M., Ellis, R. S., Taylor, K., \& Hook, R. N. 1990, MNRAS, 244, 408

Colless, M., Schade, D., Broadhurst, T. J., Ellis, R. S. 1994, MNRAS, 267, 1108

Cowie, L. L. 1991, in Observational Tests of Inflation, ed. T. Shanks, (Kluwer: Dordrecht), 25

Dalcanton, J. J. 1993, ApJ, 415, L87

Elston, R. 1994, in Infrared Astronomy with Arrays: The Next Generation, ed. I. McLean, (Dordrecht: Kluwer), 33

Fish, R. A. 1964, ApJ, 139, 284

Freeman, K. ApJ, 160, 811

Ferguson, H. C., \& McGaugh, S. S. 1995, ApJ, 440, 470

Gardner, J. L., Cowie, L. L., \& Wainscoat, R. J. 1993, ApJ, 415, L9

Glazebrook, K., Peacock, J. A., Collins, C. A., \& Miller, L. 1993 MNRAS, 266, 65

Glazebrook, K., Ellis, R., Colless, M., Broadhurst, T., Allington-Smith J., \& Tanvir, J. 1995, MNRAS, 273, 157

Gronwall, C., \& Koo, D. C., 1995, ApJ, 440, L1

Table 1: U-band counts 
Guhathakurta, P., Tyson, J. A., \& Majewski, S. R. 1990 in Evolution of the Universe of Galaxies - The Edwin Hubble Centennial Symposium, ed. R. G. Kron (San Francisco: ASP), 304

Gunn, J. E., \& Knapp, G. R. 1993, in Sky Surveys: Protostars to Protogalaxies, ed. B. T. Soifer (ASP Conf. Ser., 43), 267

Hall, P., \& Mackay, C. D. 1984, MNRAS, 210, 979

Heydon-Dumbleton, N. H., Collins, C. A., \& MacGillivray, H. T. 1989, MNRAS, 238, 379

Hintzen, P., Romanishin, W., \& Valdes, F. 1991, ApJ, 366, 7

Hubble, E. 1936, ApJ, 84, 517

Impey, C. D. 1993, in Sky Surveys: Protostars to Protogalaxies, ed. B. T. Soifer (ASP Conf. Ser., 43), 145

Jones, L. R., Fong, R., Shanks, T., Ellis, R. S., Peterson, B. A. 1991, MNRAS, 249, 481

Koo, D. C. 1986, ApJ, 311, 651

Koo, D. C., Gronwall, C., \& Bruzual A., G., ApJ, 415, L21

Koo, D. C, \& Kron, R. G. 1992, ARA\&A, 30, 613

Larson, R. B. \& Tinsley, B. M. 1978, ApJ, 381, 14

Lilly, S. J. 1993, ApJ, 411, 501

Lilly, S. J., Cowie, L. L., \& Gardner, J. P., 1991, ApJ, 369, 79

Loveday, J., Peterson, B. A., Efstathiou, G., \& Maddox, S.J. 1992, ApJ, 390, 338

Maddox, S. J., Efstathiou, G., \& Sutherland, W. J. 1990a, MNRAS, 246, 433

Maddox, S. J., Sutherland, W. J., Efstathiou, G., Loveday, J., Peterson, B. A. 1990b, MNRAS, $247,1 \mathrm{P}$

Majewski, S. R., 1989, in The Epoch of Galaxy Formation, ed. C. S. Frenk, R. S. Ellis, T. Shanks, A. F. Heavens, \& J. A. Peacock (Dordrecht: Kluwer), 85

Maoz D. \& Rix H.-W. 1993, ApJ, 416, 425

Marzke, R. O., Huchra, J. P., \& Geller, M. J. 1994, ApJ, 428, 43

McGaugh, S. S. 1992, PhD thesis, University of Michigan

Table 2: $\mathrm{B}_{J}$-band counts 
McGaugh, S. S. 1994, Nature, 367, 538

McGaugh, S. S., \& Bothun, G. D. 1994, AJ, 107, 530

McLeod, B. A., Bernstein, G. M., Rieke, M. J., Tollestrup, E. V. , Fazio, G. G. 1995, ApJS, 96, 117

Metcalfe, N., Fong, R., Shanks, T., \& Kilkenny, D. 1989, MNRAS, 236, 207

Metcalfe, N., Shanks, T., Fong, R., \& Jones, L. R. 1991, MNRAS, 249, 498

Mobasher, B., Ellis, R. S., \& Sharples, R. M. 1986, MNRAS, 223, 11

Neuschaefer, L. W., Windhorst, R. A., \& Dressler, A., 1991, ApJ, 382, 32

Peterson, B. A., Ellis, R. S., Kibblewhite, E. J., Bridgeland, M. T., Hooley, T., \& Horne, D. 1979, ApJ, 233, L109

Picard, A. 1991, AJ, 102, 445

Rieke, G. H., Loken, K., Rieke, M. J., \& Tamblyn, P. 1993, ApJ, 412, 99

Salpeter, E. E. 1955, ApJ, 121, 161

Sandage, A. 1961, ApJ, 134, 916

Sandage, A. 1988, ARA\&A, 26, 561

Schechter, P., 1976 ApJ, 203, 297

Sebok, W. K. 1986, ApJS, 62, 301

Shanks, T. 1990, in Galactic and Extragalactic Background Radiation, ed. S. Boyer \& C. Leinert (Dordrecht: Reidel), 269

Songaila, A., Cowie, L. L., Hu, E. M., \& Gardner, J. P. 1994, ApJS, 94, 461

Sprayberry, D. 1994, Ph.D. dissertation, University of Arizona

Stevenson, P. R. F, Shanks, T., \& Fong, R. 1986, in Spectral Evolution of Galaxies, ed. C. Chiosi, \& A. Renzini (Dordrecht: Reidel), 439

Thuan T. X. \& Gunn, J. E. 1976, PASP, 88, 543

Tresse, L., Hammer, F., Le Fèvre, O., \& Proust, D. 1993, A\&A, 277, 53

Tyson, J. A., 1988, AJ, 96, 1

Table 3: r-band counts 
van der Kruit, P. C. 1987, A\&A, 173, 59

Weir, N., Djorgovski, S. G., \& Fayyad, U. 1995, AJ, in press

Wyse, R. F. G., \& Silk, J. 1987, ApJ, 313, L11

Yee, H. K. C., Green, R. F., \& Stockman, H. S. 1986, ApJS, 62, 681

Yoshii, Y. 1993, ApJ, 403, 552 
Table 5: K-band counts

Table 6: Quiescent population luminosity functions

Table 7: Burst characteristics for $0.1-125 M_{\odot} \mathrm{IMF}$

Table 8: Surface brightness selection criteria 


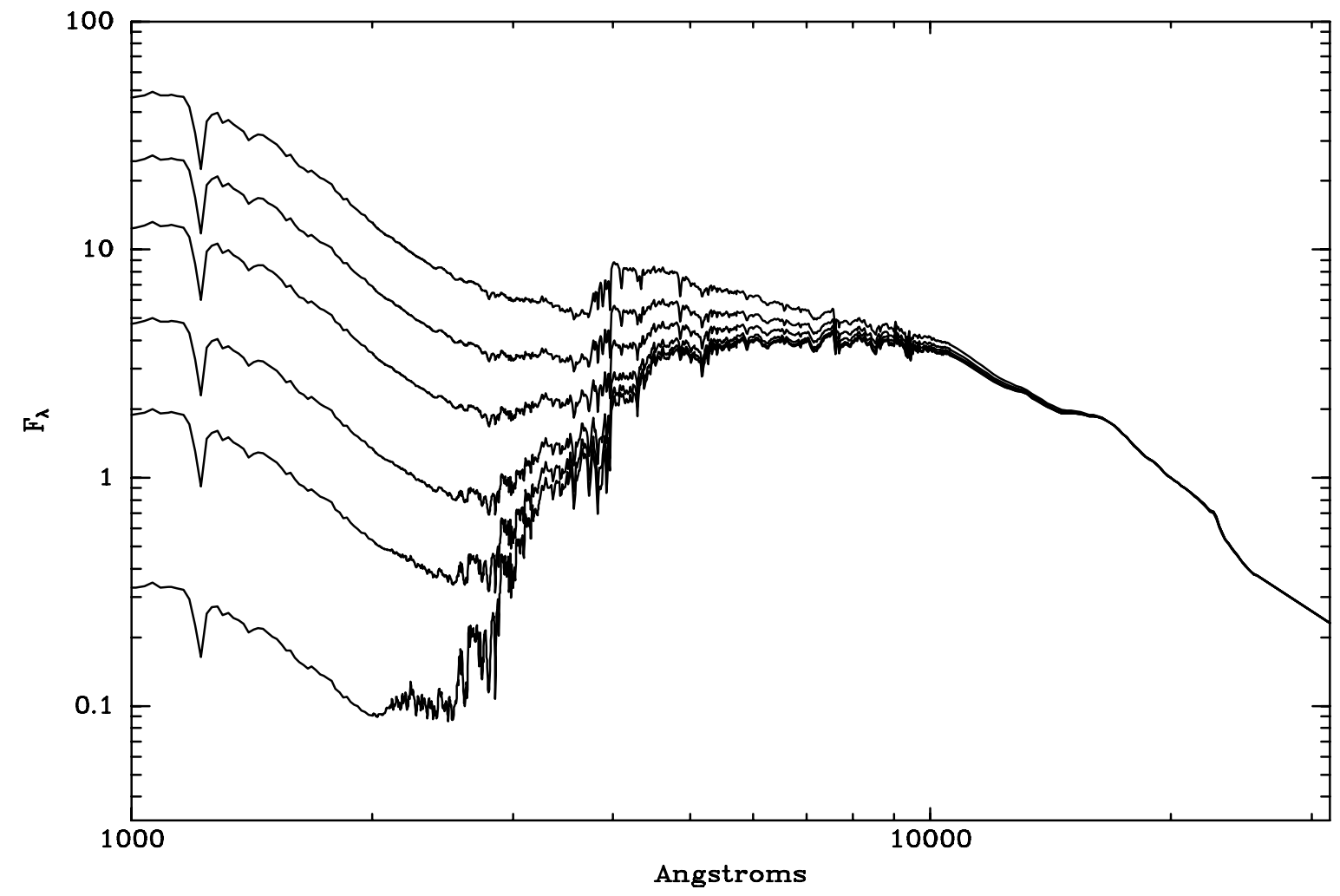

Fig. 1.-

Galaxy spectral energy distributions at an age of 13.5 Gyr generated by Bruzual \& Charlot (1993) galaxy evolution models. From top to bottom, the constant star-formation rate per Gyr relative to the amount of star formation in the initial burst is $1.0,0.03,0.01,0.003,0.001$, and 0.00001 . 


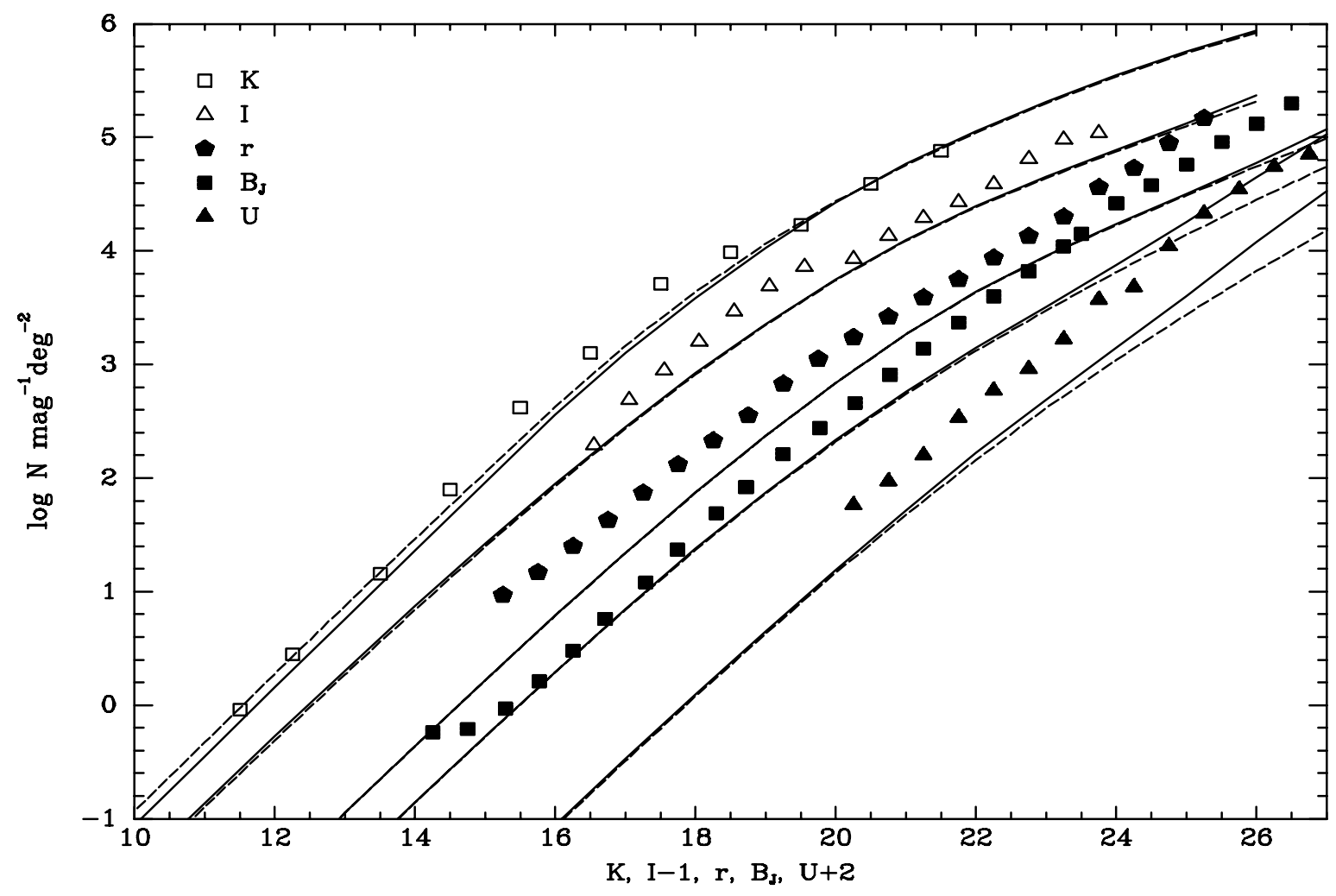

Fig. 2.-

Comparison of expected counts using non-evolving Bruzual SEDs (solid) vs Coleman et al. SEDs (dashed). The biggest differences occur in the faint $B_{J}$ and $U$ counts where redshifted UV flux is detected. Data plotted is listed in Tables 15 . 


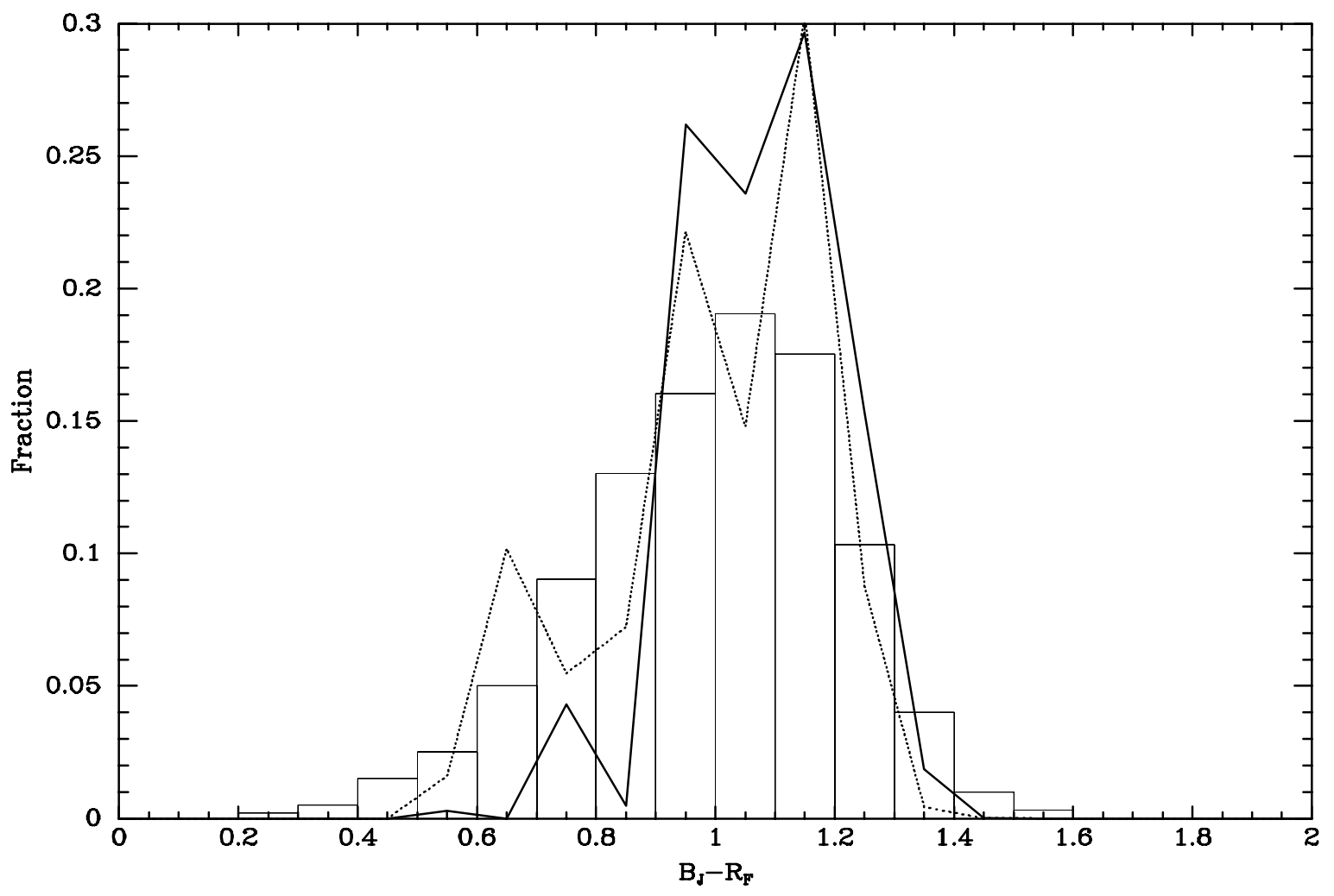

Fig. 3.-

Comparison of predicted color distributions $\left(B_{J}-R_{F}\right)$ for bright galaxies $\left(15<B_{J}<17\right)$ with observations. Transformations are $B_{J}=B-0.3(B-V)$ and $R_{F}=R-0.06(B-R)$. The histogram is observations from Koo \& Kron (1992). The solid line shows the Lilly (1993) type-dependent LF. The dashed line shows the Shanks color-dependent LF. We prefer the Shanks LF because the colors match the observations better. 


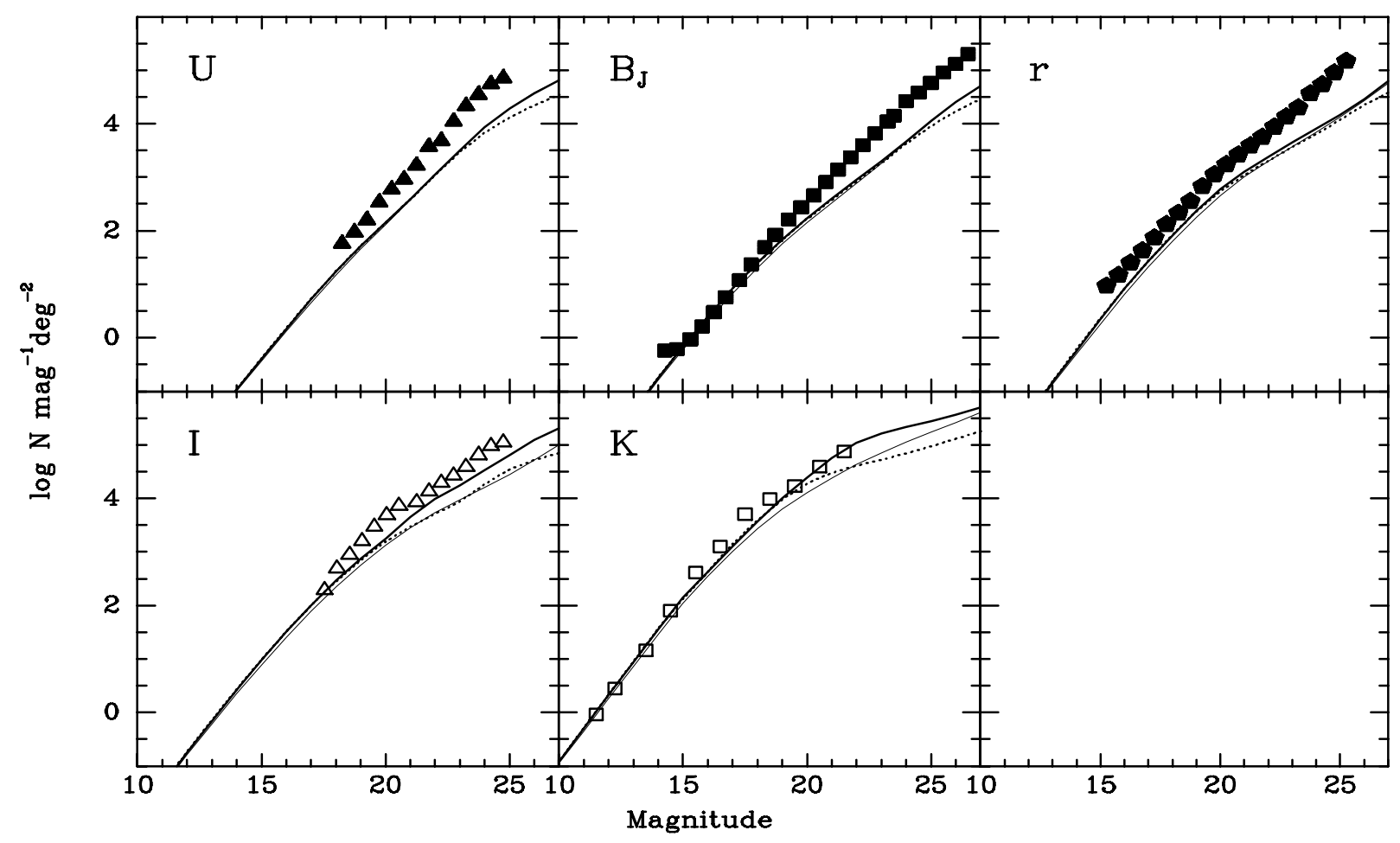

Fig. 4.-

Galaxy numbers vs. magnitude for simple models. The thin line shows the unphysical non-evolving model. The solid line is the passively evolving population with $q_{0}=0.05$ and $H_{0}=60 \mathrm{~km} \mathrm{~s}^{-1} \mathrm{Mpc}^{-1}$. The dotted line is the same with $q_{0}=0.5$ and $H_{0}=47 \mathrm{~km} \mathrm{~s}^{-1} \mathrm{Mpc}^{-1}$. The redshift of galaxy formation is $\mathrm{z}=7.3$ in both cases. 


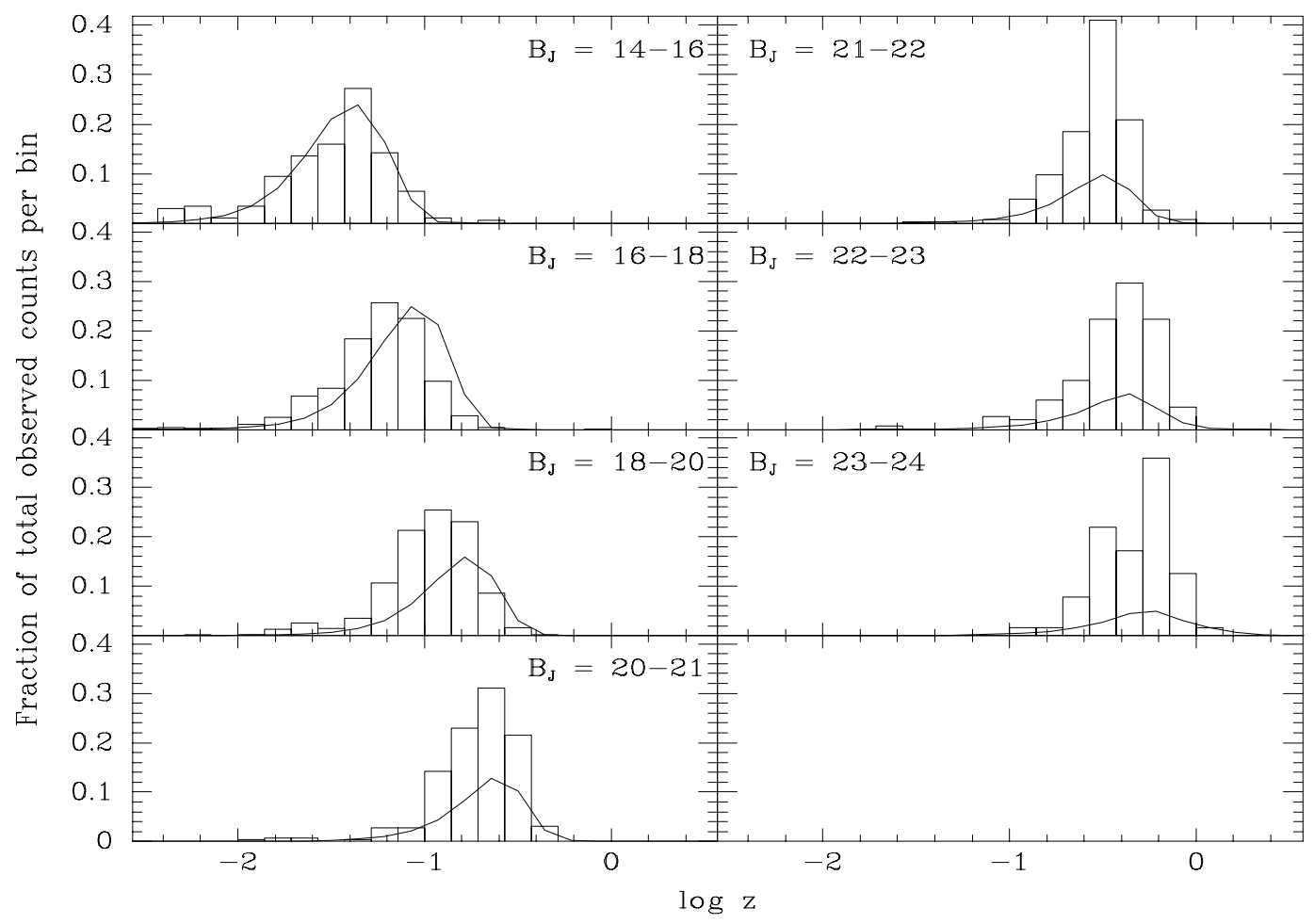

Fig. 5.-

Redshift distributions for the baseline passively evolving model $\left(q_{0}=0.05, H_{0}=60 \mathrm{~km} \mathrm{~s}^{-1} \mathrm{Mpc}^{-1}\right.$, $t=13.5 \mathrm{Gyr})$. The solid line is the model prediction. The histograms are the observations presented in Koo et al. (1993). The models are not renormalized; thus, the ratio of the number of predicted galaxies at each magnitude relative to the number observed (Figure 14) is preserved. 


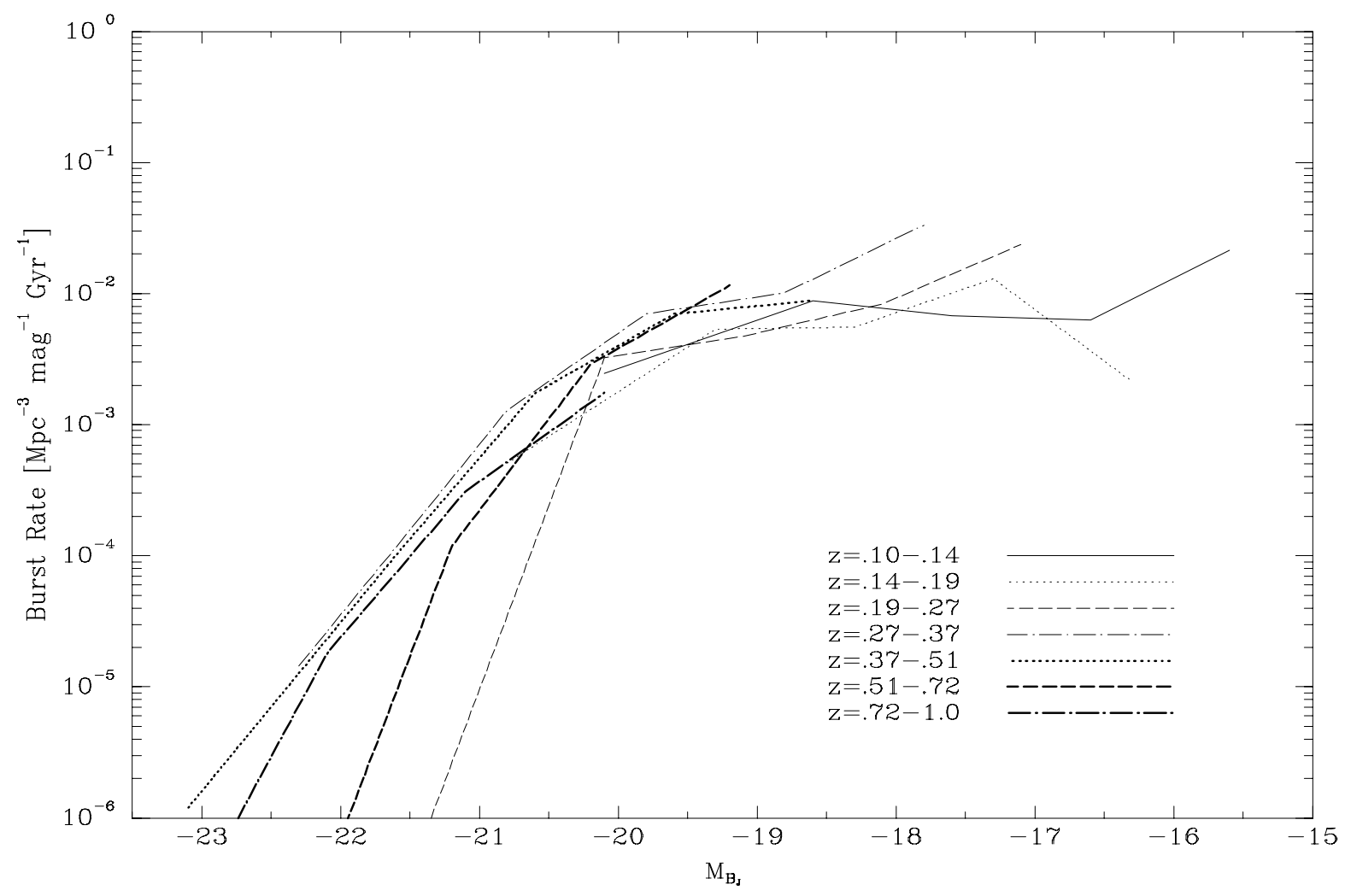

Fig. 6.-

Derived burst rate luminosity functions for 0.1 Gyr burst populations. The IMF is Salpeter for $0.1-125 M_{\odot} ; H_{0}=50 \mathrm{~km} \mathrm{~s}^{-1} \mathrm{Mpc}^{-1}$. The horizontal axis is the peak $B_{J}$ absolute magnitude. The vertical axis is scaled in terms of the number of galaxies that reach the specified peak magnitude per unit comoving volume per Gyr. 


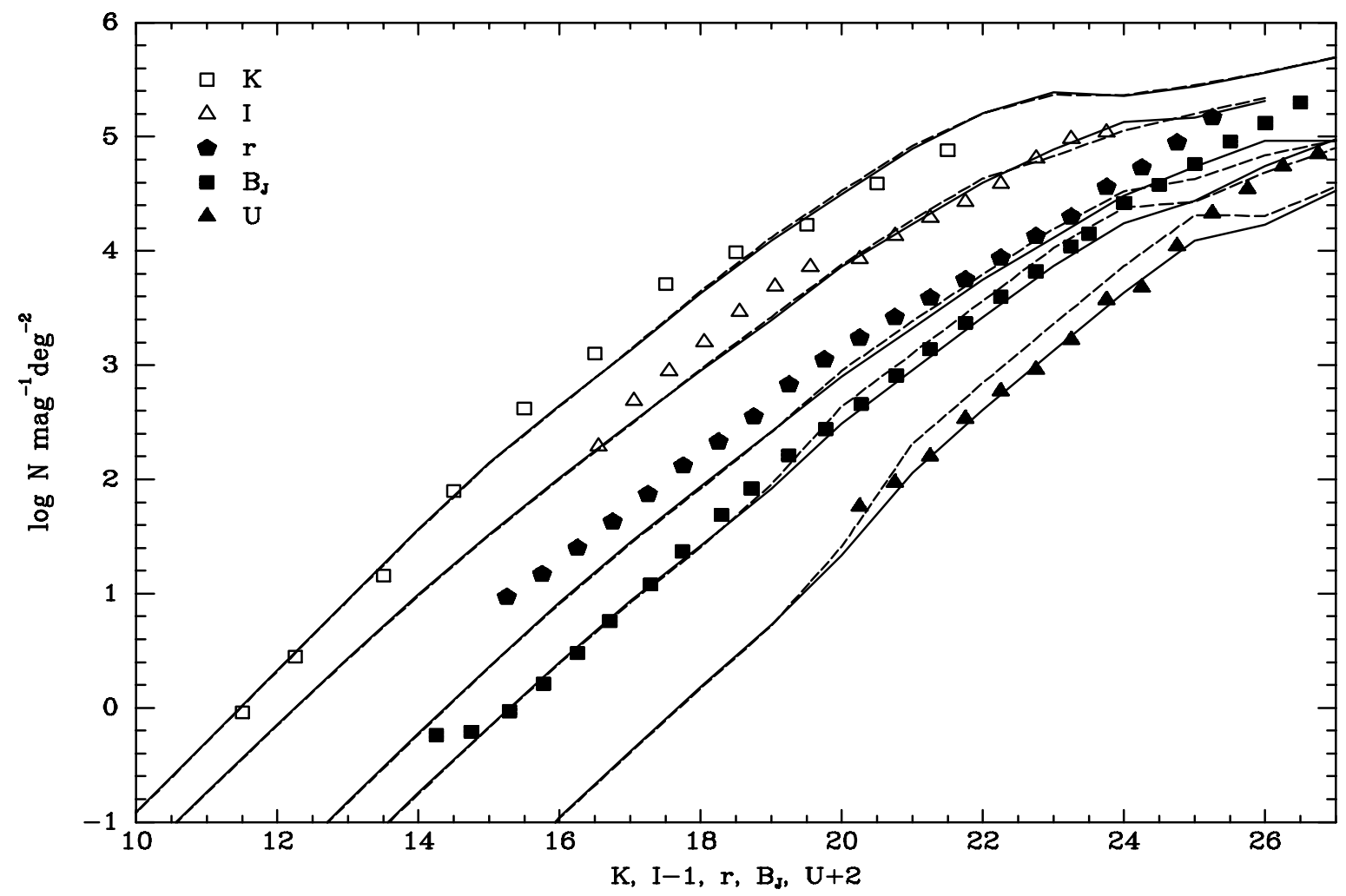

Fig. 7.-

Number vs. magnitude for burst populations. Solid line is for $0.1-125 M_{\odot}$ IMF. Dashed line is for $2.5-125 M_{\odot} \mathrm{IMF}$. 


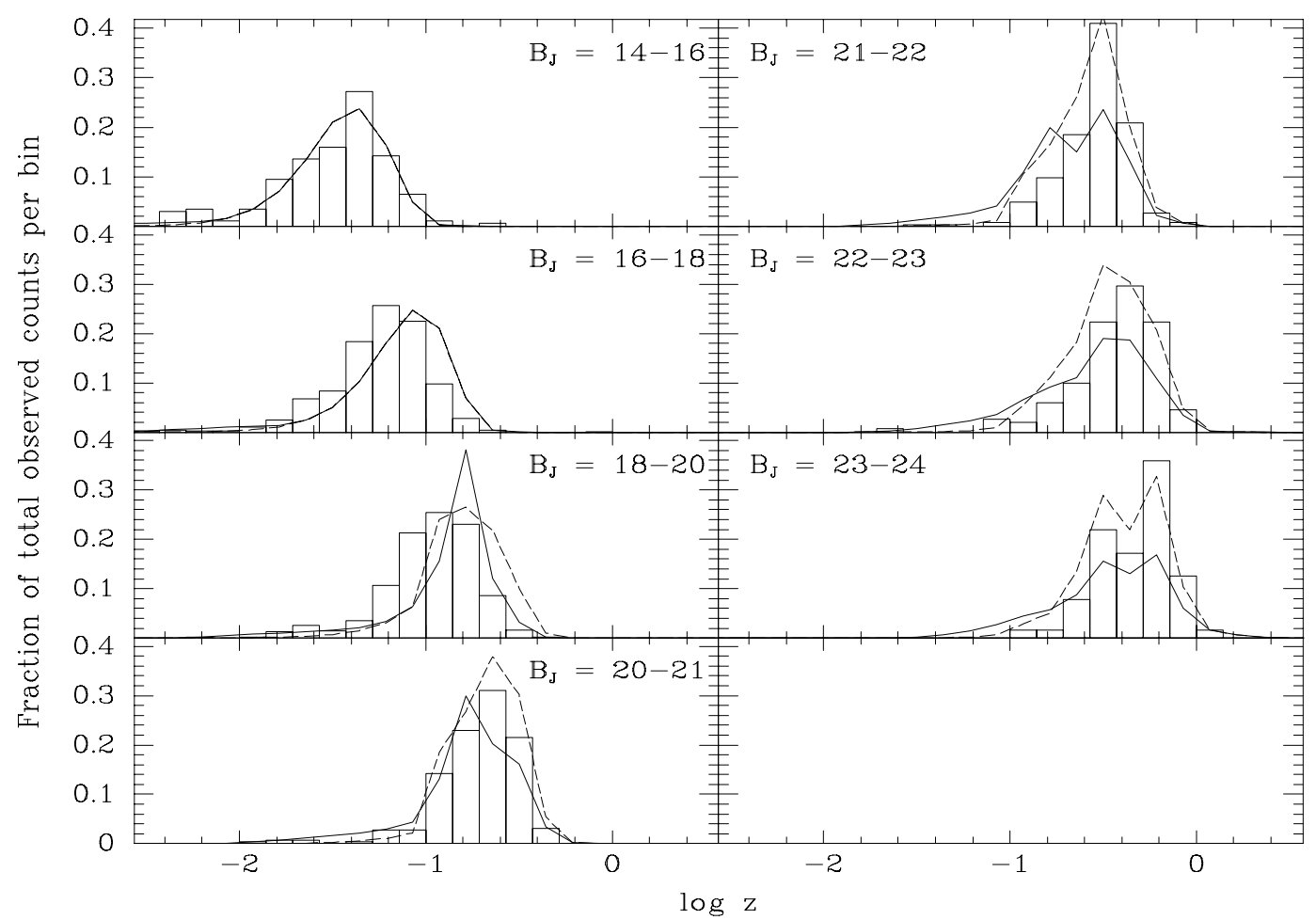

Fig. 8.-

Redshift distributions for burst populations. Solid line is for $0.1-125 M_{\odot}$ IMF. Dashed line is for $2.5-125 M_{\odot}$ IMF. 


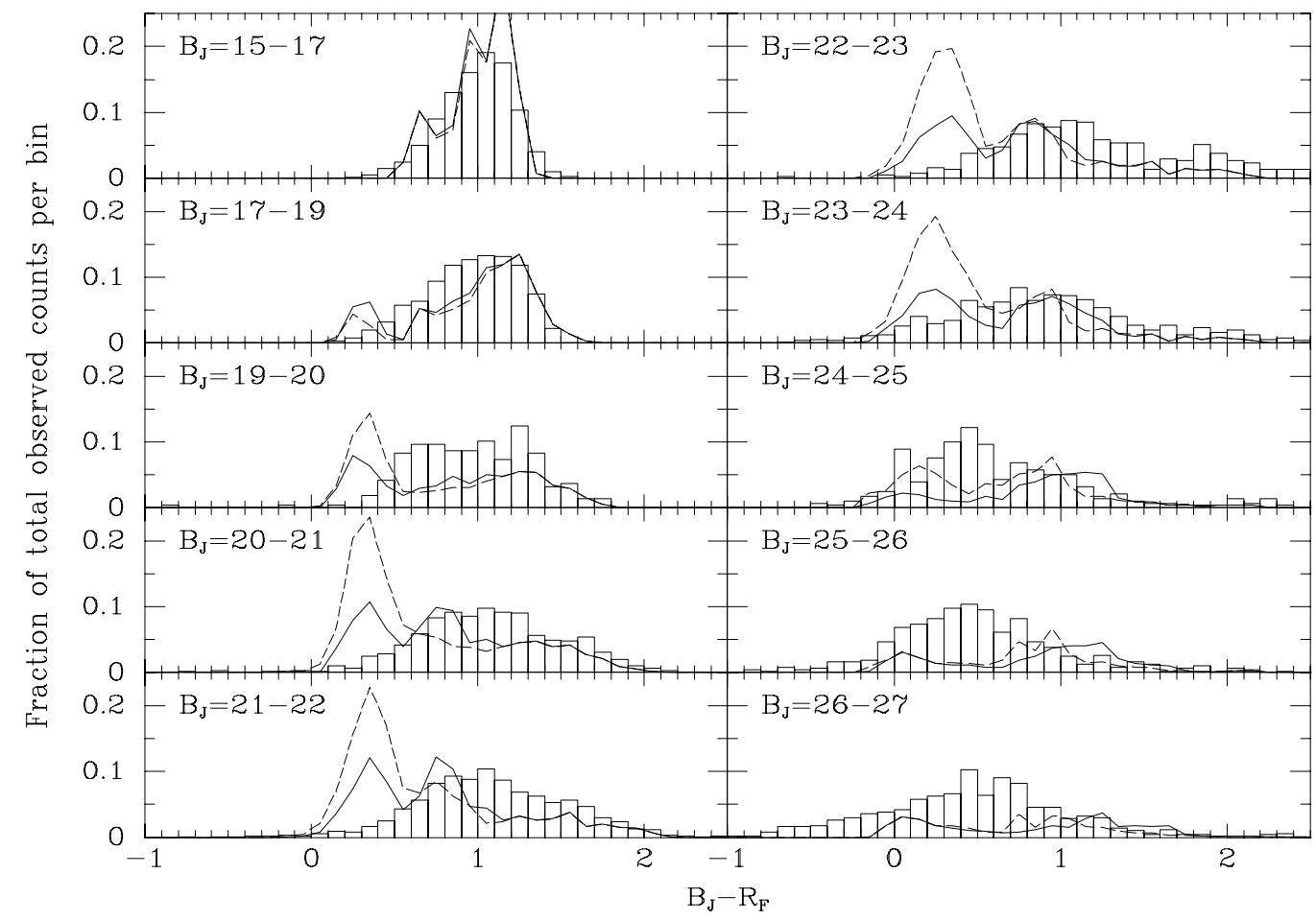

Fig. 9.-

$B_{J}-R_{F}$ color distributions for burst populations. Solid line is for $0.1-125 M_{\odot}$ IMF. Dashed line is for $2.5-125 M_{\odot} \mathrm{IMF}$. 


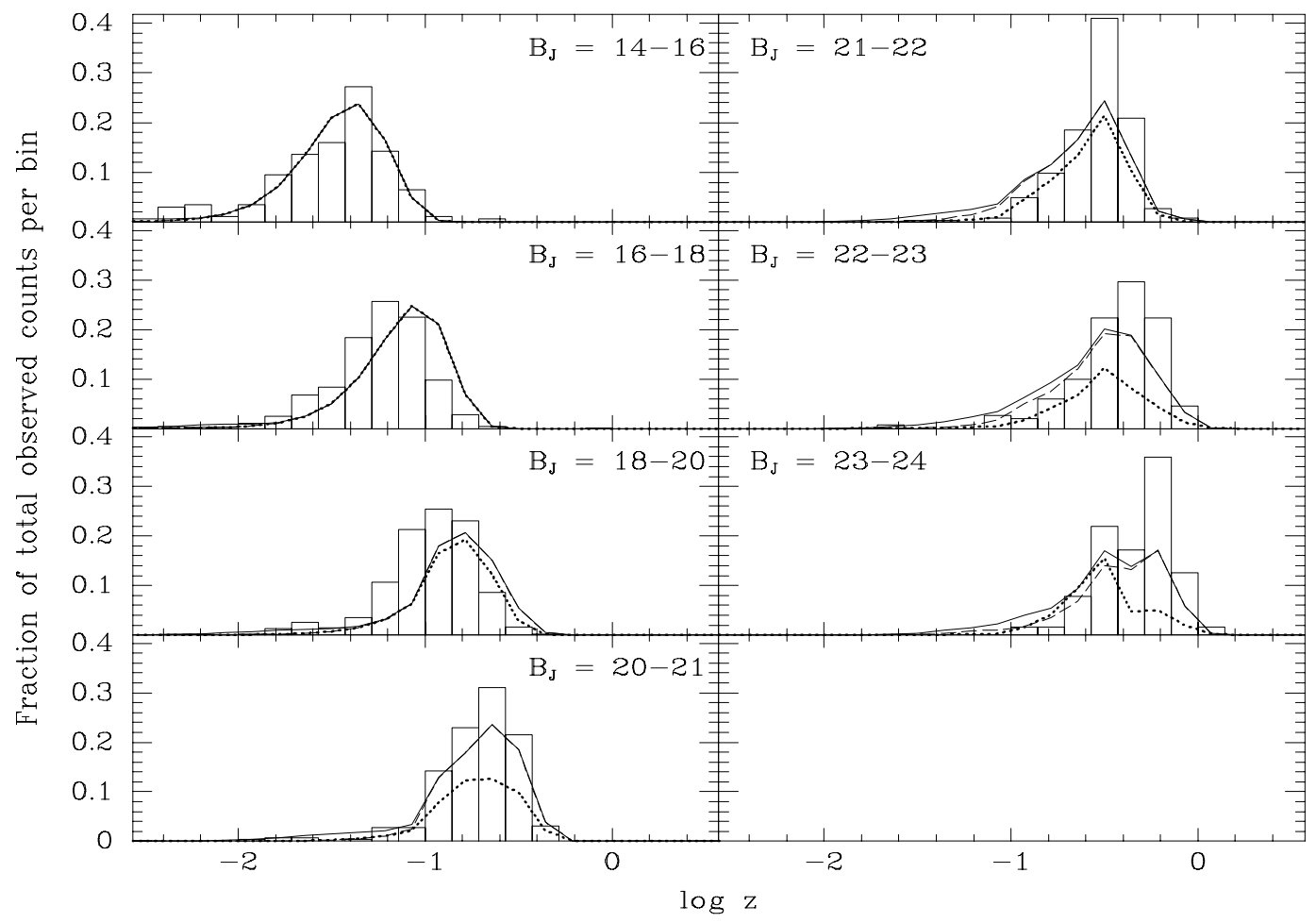

Fig. 10.-

$B_{J}$ selected redshift distributions incorporating surface brightness selection for the baseline plus local IMF burst populations. The solid, dashed and dotted curves are for populations where a $10^{8} M_{\odot}$ burst has $r_{e}=1,3$, and $10 \mathrm{kpc}$ respectively $\left(H_{0}=50 \mathrm{~km} \mathrm{~s}^{-1} \mathrm{Mpc}^{-1}, q_{0}=0.05\right)$. The three curves are degenerate in the upper-left plots because the burst population does not contribute at those magnitudes. 


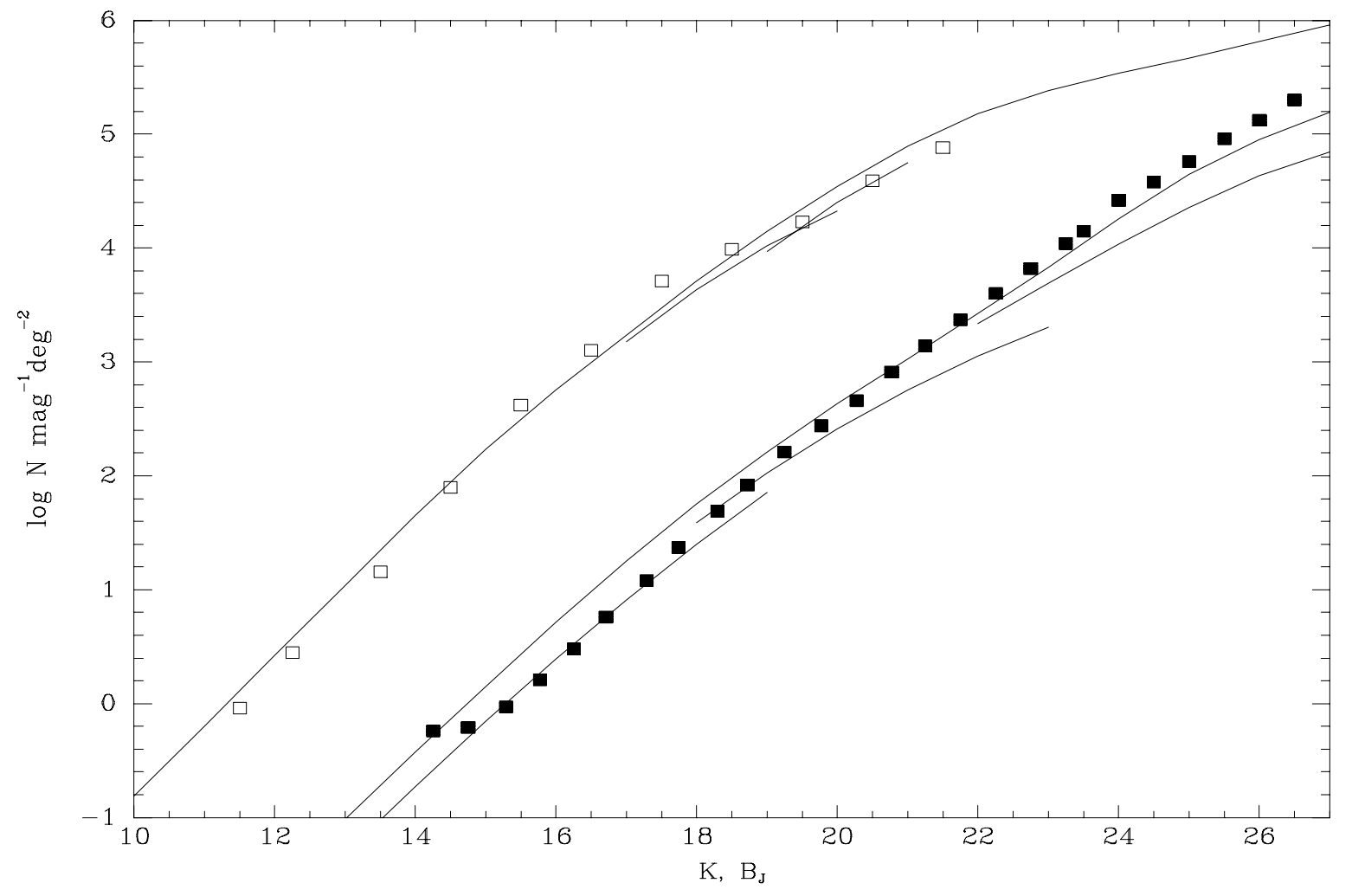

Fig. 11.-

Number vs. magnitude for a baseline population plus an equivalent number of LSB galaxies. The long-upper curves are for no surface brightness selection. The lower 3 curves show what should actually be observed using the selection criteria that are listed in Table 8. The predictions match the observations fairly well for $B_{J} \lesssim 23$. 


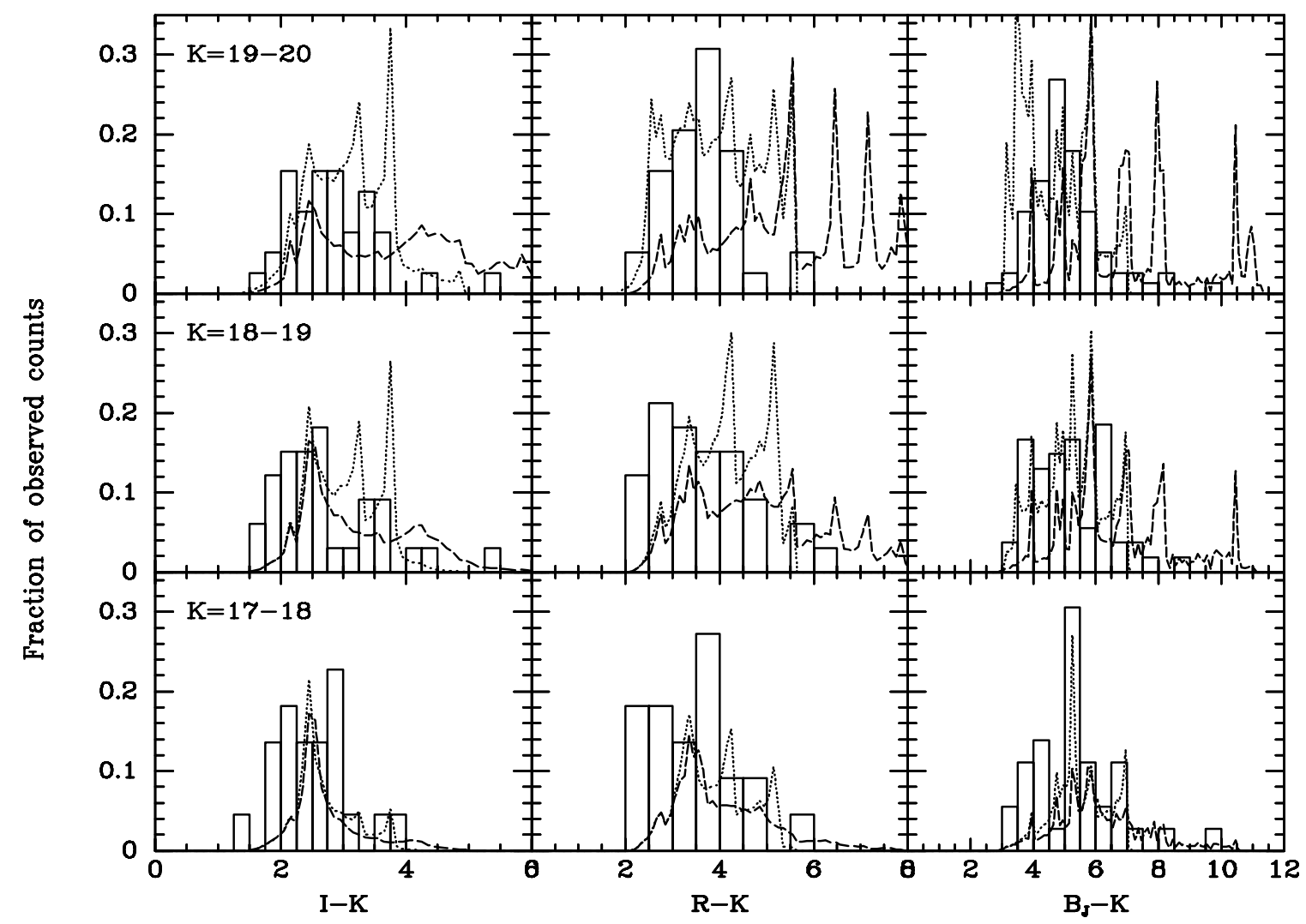

Fig. 12.-

Colors of $K$-selected galaxies. The $K$-magnitude range is shown in the left panel of each row. The histogram shows data from McLeod et al. (1995). Dashed line is for the baseline population with constant SFR. Dotted line is for the modified population with exponential SFR. 


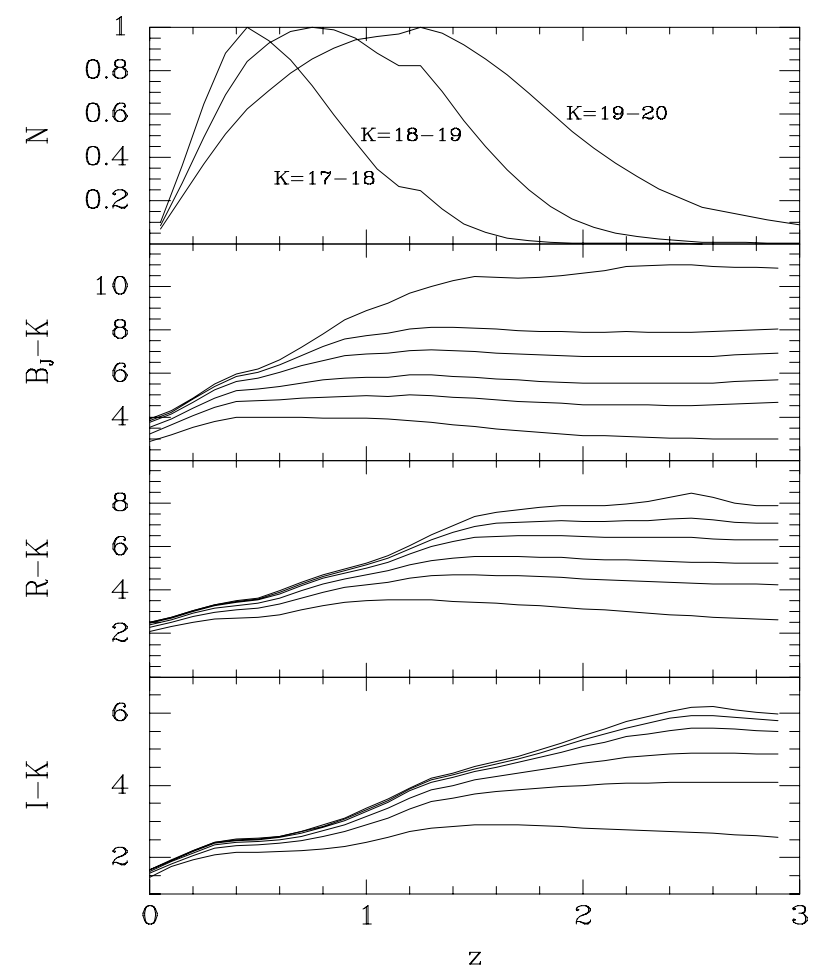

Fig. 13.-

The bottom three panels show the colors of the evolving baseline population as a function of redshift. The six curves are for the E though Im types from top to bottom. The upper panel shows the predicted redshift distributions for a $K$-selected population. From left to right the three curves are for $K=17-18,18-19$ and $19-20$. 


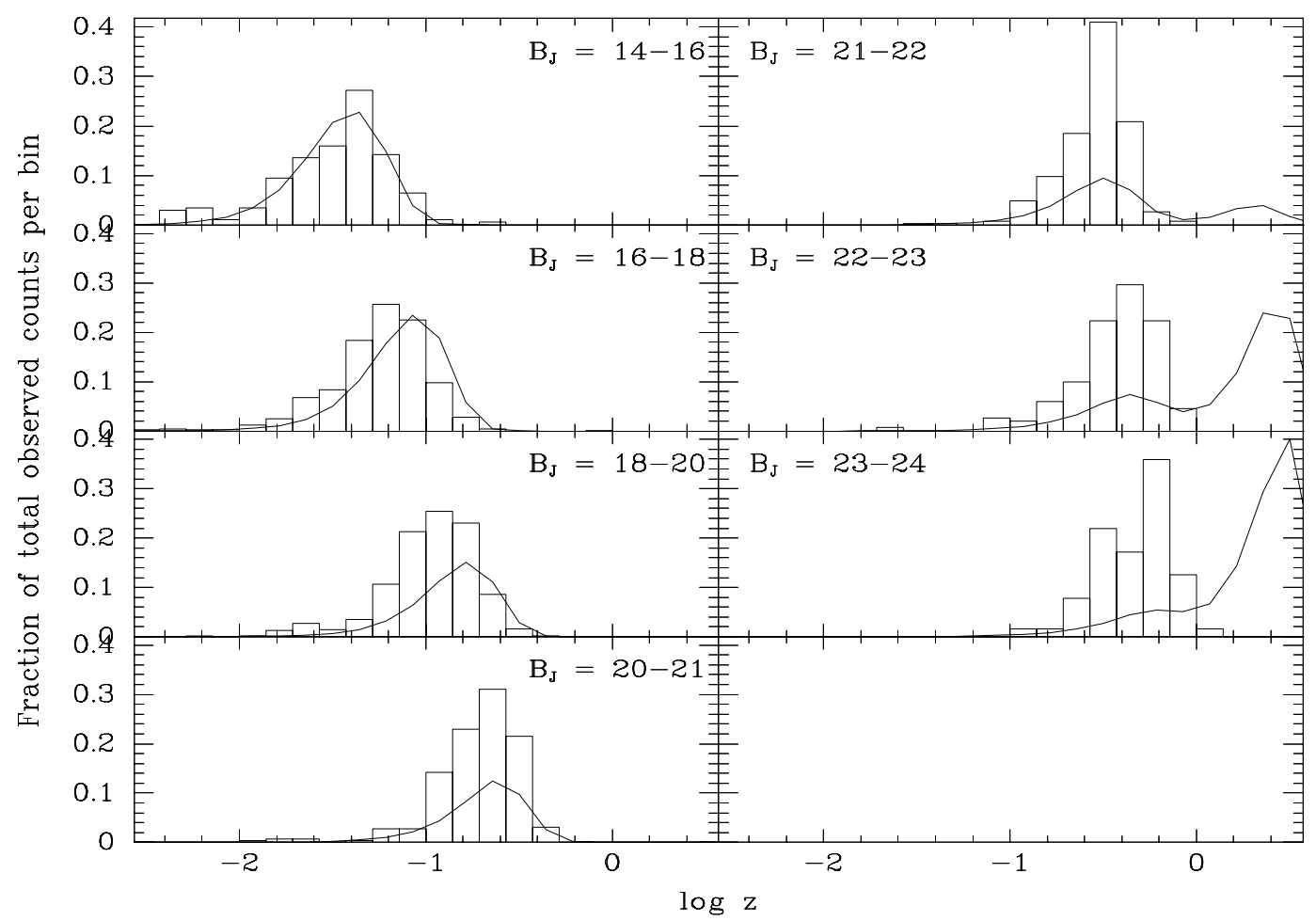

Fig. 14.-

$B_{J}$-selected redshift distributions for the exponential modified baseline population. The increased evolution of the red galaxies adds too many galaxies in the blue. For this model to work, dust must extinguish the blue light. 
TABLE 1

U-BAND COUNTS

\begin{tabular}{llrr}
\hline \hline$U$ & $\log n\left[\mathrm{mag}^{-1} \mathrm{deg}^{-2}\right]$ & $N$ & Ref \\
\hline 18.25 & 1.76 & 31 & 1,2 \\
18.75 & 1.97 & 44 & 1,2 \\
19.25 & 2.20 & 70 & 1,2 \\
19.75 & 2.53 & 150 & 1,2 \\
20.25 & 2.77 & 260 & 1,2 \\
20.75 & 2.96 & 540 & 1,2 \\
21.25 & 3.22 & 420 & 1 \\
21.75 & 3.57 & 920 & 1 \\
22.25 & 3.68 & 170 & 3,4 \\
22.75 & 4.04 & 140 & 3,4 \\
23.25 & 4.33 & 280 & 3,4 \\
23.75 & 4.54 & 450 & 3,4 \\
24.25 & 4.74 & 720 & 3,4 \\
24.75 & 4.85 & 530 & 4 \\
& & & \\
\hline
\end{tabular}

References.- (1) Koo (1986); (2) Jones et al. (1991); (3) Majewski (1989); (4) Guhathakurta et al. (1990). 
TABLE 2

$\mathrm{B}_{J}$-BAND COUNTS

\begin{tabular}{lrrr}
\hline \hline \multicolumn{3}{c}{$B \log n\left[\mathrm{mag}^{-1} \mathrm{deg}^{-2}\right]$} & \multicolumn{1}{r}{ Ref } \\
\hline 14.25 & -0.24 & 55 & 1,2 \\
14.75 & -0.21 & 60 & 1,2 \\
15.29 & -0.03 & 800 & 3 \\
15.77 & 0.21 & 1400 & 3 \\
16.25 & 0.48 & 2600 & 3 \\
16.71 & 0.76 & 4900 & 3 \\
17.29 & 1.08 & 10000 & 3 \\
17.74 & 1.37 & 20000 & 3 \\
18.29 & 1.69 & 42000 & 3 \\
18.72 & 1.92 & 71000 & 3 \\
19.25 & 2.21 & 140000 & 3 \\
19.77 & 2.44 & 240000 & 3 \\
20.27 & 2.66 & 400000 & 3 \\
20.77 & 2.91 & 690000 & 3 \\
21.25 & 3.14 & 2200 & 4 \\
21.75 & 3.37 & 3800 & 4 \\
22.25 & 3.60 & 6400 & 4 \\
22.75 & 3.82 & 11000 & 4 \\
23.25 & 4.04 & 17500 & 4 \\
23.5 & 4.15 & 2000 & $5,6,7$ \\
24.0 & 4.42 & 3800 & $5,6,7$ \\
24.5 & 4.58 & 5100 & $5,6,7$ \\
25.0 & 4.76 & 2600 & 5 \\
25.5 & 4.96 & 3600 & 5,8 \\
26.0 & 5.12 & 4100 & 5,8 \\
26.5 & 5.30 & 120 & 8 \\
& & & \\
\hline & & &
\end{tabular}

References.- (1) Ciardullo (1987); (2) Heydon-Dumbleton et al. (1989); (3) Maddox et al. (1990); (4) Jones et al. (1991); (5) Tyson (1988); (6) Metcalfe et al. (1991); (7) Neuschaefer et al. (1991); (8) Lilly et al. (1991). 
TABLE 3

R-BAND COUNTS

\begin{tabular}{lcrr}
\hline \hline \multicolumn{2}{c}{$r \log n\left[\mathrm{mag}^{-1} \mathrm{deg}^{-2}\right] N$} & \multicolumn{1}{c}{ Ref } \\
\hline 15.25 & 0.97 & 1800 & 2,3 \\
15.75 & 1.17 & 3000 & $1,2,3$ \\
16.25 & 1.40 & 5200 & $1,2,3$ \\
16.75 & 1.63 & 8800 & $1,2,3$ \\
17.25 & 1.87 & 15000 & $1,2,3$ \\
17.75 & 2.12 & 27000 & $1,2,3$ \\
18.25 & 2.33 & 42000 & 2,3 \\
18.75 & 2.55 & 69000 & 2,3 \\
19.25 & 2.83 & 1400 & 1,8 \\
19.75 & 3.05 & 2400 & 1,8 \\
20.25 & 3.24 & 3700 & 1,8 \\
20.75 & 3.42 & 5600 & 1,8 \\
21.25 & 3.59 & 8300 & 1,8 \\
21.75 & 3.75 & 12000 & 1,8 \\
22.25 & 3.94 & 660 & $4,5,6,7$ \\
22.75 & 4.13 & 930 & $4,6,7$ \\
23.25 & 4.30 & 450 & 4,6 \\
23.75 & 4.56 & 830 & 4,6 \\
24.25 & 4.73 & 1200 & 4,6 \\
24.75 & 4.95 & 1500 & 6 \\
25.25 & 5.17 & 1900 & 6 \\
& & & \\
\hline
\end{tabular}

References.- (1) Stevenson et al. (1986); (2) Sebok (1986); (3) Picard (1991); (4) Hall \& Mackay (1984); (5) Yee et al. (1986); (6) Tyson (1988); (7) Metcalfe et al. (1991); (8) Jones et al. (1991). 
TABLE 4

I-BAND COUNTS

\begin{tabular}{lcrr}
\hline \hline$I \quad \log n\left[\mathrm{mag}^{-1} \mathrm{deg}^{-2}\right]$ & & Ref \\
\hline 17.55 & 2.29 & 21 & 1 \\
18.05 & 2.69 & 52 & 1 \\
18.55 & 2.95 & 220 & 1 \\
19.05 & 3.20 & 400 & 1 \\
19.55 & 3.47 & 700 & 1 \\
20.05 & 3.69 & 1200 & 1 \\
20.55 & 3.86 & 1800 & 1 \\
21.25 & 3.93 & 320 & 2,3 \\
21.75 & 4.13 & 500 & 2,3 \\
22.25 & 4.29 & 440 & 4,2 \\
22.75 & 4.43 & 610 & 4,2 \\
23.25 & 4.59 & 850 & 4,2 \\
23.75 & 4.81 & 1200 & 2,5 \\
24.25 & 4.98 & 1700 & 2,5 \\
24.75 & 5.04 & 31 & 5 \\
& & & \\
\hline
\end{tabular}

References.- (1) Koo (1986); (2) Tyson (1988); (3) Hintzen et al (1991); (4) Hall \& Mackay (1984); (5) Lilly et al. (1991). 
TABLE 5

K-BAND COUNTS

\begin{tabular}{lrrr}
\hline \hline$K$ & $\log n\left[\mathrm{mag}^{-1} \mathrm{deg}^{-2}\right]$ & $N$ & Ref \\
\hline 11.5 & -0.04 & 32 & 1 \\
12.25 & 0.45 & 49 & 1 \\
13.5 & 1.16 & 23 & 2 \\
14.5 & 1.90 & 146 & 2,3 \\
15.5 & 2.62 & 155 & 2,3 \\
16.5 & 3.10 & 491 & 2,3 \\
17.5 & 3.71 & 232 & 2,4 \\
18.5 & 3.99 & 207 & 2,4 \\
19.5 & 4.23 & 158 & 2,4 \\
20.5 & 4.59 & 104 & 2,4 \\
21.5 & 4.88 & 74 & 2,4 \\
& & & \\
\hline
\end{tabular}

References.- (1) Mobasher et al. (1986); (2) Gardner et al (1993); (3) Glazebrook et al (1993); (4) McLeod et al (1994).

TABLE 6

QUIESCENT POPULATION LUMINOSITY FUNCTIONS

\begin{tabular}{|c|c|c|c|c|c|}
\hline \multirow[t]{2}{*}{ Type } & \multirow[t]{2}{*}{$\mathrm{SFR}^{\mathrm{a}}$} & \multirow[t]{2}{*}{$B-V$} & \multicolumn{3}{|c|}{ Schechter parameters ${ }^{b}$} \\
\hline & & & $\phi^{*}\left[\mathrm{Mpc}^{-3} \mathrm{mag}^{-1}\right]$ & $M_{B_{J}}^{*}$ & $\alpha$ \\
\hline E... & 0.00001 & 0.94 & $1.92 \times 10^{-4}$ & -21.1 & -0.7 \\
\hline So $\ldots \ldots \ldots \ldots$ & 0.001 & 0.90 & $1.92 \times 10^{-4}$ & -21.1 & -0.7 \\
\hline Sab........... & 0.003 & 0.83 & $1.92 \times 10^{-4}$ & -21.1 & -0.7 \\
\hline $\mathrm{Sc} \ldots \ldots \ldots \ldots$ & 0.01 & 0.7 & $2.60 \times 10^{-4}$ & -21.4 & -1.1 \\
\hline $\mathrm{Sdm} .$. & 0.03 & 0.57 & $0.85 \times 10^{-4}$ & -21.5 & -1.5 \\
\hline $\operatorname{Im} \ldots$ & 1.0 & 0.42 & $0.85 \times 10^{-4}$ & -21.5 & -1.5 \\
\hline
\end{tabular}

${ }^{a}$ Constant SFR $\mathrm{Gyr}^{-1}$ relative to initial burst

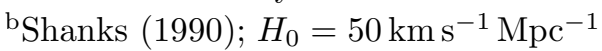


TABLE 7

BURST CHARACTERISTICS FOR 0.1-125M $\odot$ IMF

\begin{tabular}{|c|c|c|c|}
\hline Redshift & $\begin{array}{c}\text { Present } \\
\text { Age }(\mathrm{Gyr})\end{array}$ & $\Delta M^{\mathrm{a}}$ & $\mu^{\mathrm{b}}$ \\
\hline $0.10-0.14 \ldots \ldots \ldots$ & 2.0 & 4.3 & 26.1 \\
\hline $0.14-0.19 \ldots \ldots \ldots$ & 2.6 & 4.7 & 26.4 \\
\hline $0.19-0.27 \ldots \ldots \ldots$ & 3.4 & 5.0 & 26.7 \\
\hline $0.27-0.37 \ldots \ldots \ldots$ & 4.3 & 5.2 & 26.9 \\
\hline $0.37-0.51 \ldots \ldots \ldots$ & 5.5 & 5.5 & 27.2 \\
\hline $0.51-0.72 \ldots \ldots \ldots$ & 6.7 & 5.6 & 27.3 \\
\hline $0.72-1.00 \ldots \ldots \ldots$ & 8.0 & 5.7 & 27.4 \\
\hline
\end{tabular}

${ }^{a}$ Amount of fading between peak brightness and present.

${ }^{\mathrm{b}}$ Present day central surface brightness so that $10^{8} M_{\odot}$ burst has $r_{e}=3 \mathrm{kpc}$.

TABLE 8

SURFACE BRIGHTNESS SELECTION CRITERIA

\begin{tabular}{|c|c|c|c|c|c|c|}
\hline Filter & $\mathrm{m}$ & $\begin{array}{c}\text { FWHM } \\
{\left[{ }^{\prime \prime}\right]}\end{array}$ & $\begin{array}{c}\mu_{d e t} \\
{\left[\mathrm{mag} / \square^{\prime \prime}\right]}\end{array}$ & $\begin{array}{c}r_{\min } \\
{\left[{ }^{\prime \prime}\right]}\end{array}$ & $\begin{array}{l}\text { Magnitude } \\
\text { Type }\end{array}$ & Source \\
\hline \multicolumn{7}{|c|}{ Criteria for $n(z)$} \\
\hline$B_{J}$ & $14-18$ & $1.0^{\mathrm{a}}$ & 25.0 & $2.0^{\mathrm{a}}$ & Total & Metcalfe et al. (1989) \\
\hline$B_{J}$ & $18-21$ & $1.8^{\mathrm{a}}$ & $25.5^{\mathrm{a}}$ & $0.6^{\mathrm{a}}$ & Total $^{\mathrm{a}}$ & Koo \& Kron (1992) \\
\hline$B_{J}$ & $21-23$ & 1.8 & 26.5 & 0.6 & Total & Colless et al. (1990) \\
\hline$B_{J}$ & $23-24$ & $1.1^{\mathrm{a}}$ & 29.0 & $0.8^{\mathrm{a}}$ & $3^{\prime \prime}$ aperture & Lilly et al. (1991) \\
\hline \multicolumn{7}{|c|}{ Criteria for $n(m)$} \\
\hline$B_{J}$ & $<19$ & 1.0 & 24.7 & 1.1 & Pseudototal $^{\mathrm{b}}$ & Maddox et al. (1990b) \\
\hline$B_{J}$ & $18-23$ & 1.8 & 26.5 & 0.6 & Isophotal & Jones et al. (1991) \\
\hline$B_{J}$ & $22-27$ & 1.0 & 29.0 & 0.8 & Isophotal & Tyson (1988) \\
\hline$K$ & $17-20$ & 2.1 & 23.0 & 1.3 & Pseudototal $^{\mathrm{c}}$ & McLeod et al. (1994) \\
\hline$K$ & $19-21$ & 1.8 & 23.6 & 0.5 & $3^{\prime \prime}$ aperture & Cowie $^{\mathrm{d}}$ \\
\hline
\end{tabular}

${ }^{a}$ Estimated-not given in source.

${ }^{\mathrm{b}}$ Used correction scheme given in Maddox et al. (1990a)

${ }^{\mathrm{c}}$ Flux is measured in area double that defined by $\mu_{\text {det }}$

${ }^{\mathrm{d}}$ As presented by Yoshii (1993) 